\title{
TENSOR DECOMPOSITION, PARAFERMIONS, LEVEL-RANK DUALITY, AND RECIPROCITY LAW FOR VERTEX OPERATOR ALGEBRAS
}

\author{
CUIPO (CUIBO) JIANG AND ZONGZHU LIN
}

\begin{abstract}
For the semisimple Lie algebra $\mathfrak{s l}_{n}$, the basic representation $L_{\widehat{\mathfrak{s l}}_{n}}(1,0)$ of the affine Lie algebra $\widehat{\mathfrak{s t}_{n}}$ is a lattice vertex operator algebra. The first main result of the paper is to prove that the commutant vertex operator algebra of $L_{\widehat{\mathfrak{s}}_{n}}(l, 0)$ in the $l$ fold tensor product $L_{\widehat{\mathfrak{s l}}_{n}}(1,0)^{\otimes l}$ is isomorphic to the parafermion vertex operator algebra $K\left(\mathfrak{s l}_{l}, n\right)$, which is the commutant of the Heisenberg vertex operator algebra $L_{\widehat{\mathfrak{h}}}(n, 0)$ in $L_{\widehat{\mathfrak{s l}}_{l}}(n, 0)$. The result provides a version of level-rank duality. The second main result of the paper is to prove more general version of the first result that the commutant of $L_{\widehat{\mathfrak{s} n_{n}}}\left(l_{1}+\cdots+l_{s}, 0\right)$ in $L_{\widehat{\mathfrak{s}_{n}}}\left(l_{1}, 0\right) \otimes \cdots \otimes L_{\widehat{\mathfrak{s l}_{n}}}\left(l_{s}, 0\right)$ is isomorphic to the commutant of the vertex operator algebra generated by a Levi Lie subalgebra of $\mathfrak{s l}_{l_{1}+\cdots+l_{s}}$ corresponding to the composition $\left(l_{1}, \cdots, l_{s}\right)$ in the rational vertex operator algebra $L_{\widehat{\mathfrak{s}}_{l_{1}+\cdots+l_{s}}}(n, 0)$. This general version also resembles a version of reciprocity law discussed by Howe in the context of reductive Lie groups. In the course of the proof of the main results, certain Howe duality pairs also appear in the context of vertex operator algebras.
\end{abstract}

\section{IntRODUCTION}

1.1. Given a finite dimensional simple Lie algebra $\mathfrak{g}$, let $\widehat{\mathfrak{g}}=\mathfrak{g} \otimes\left[t, t^{-1}\right] \oplus \mathbb{C} K$ be its affine Lie algebra. Let $L_{\mathfrak{\mathfrak { g }}}(1,0)$ be the basic representation of $\widehat{\mathfrak{g}}$. Then for any $l \in \mathbb{N}$, the tensor product $\widehat{\mathfrak{g}}$-module $L_{\widehat{\mathfrak{g}}}(1,0)^{\otimes l}$ is a direct sum of irreducible $\widehat{\mathfrak{g}}$-modules and there is a decomposition

$$
L_{\widehat{\mathfrak{g}}}(1,0)^{\otimes l}=\bigoplus L_{\widehat{\mathfrak{g}}}(l, \bar{\Lambda}) \otimes_{\mathbb{C}} M_{\widehat{\mathfrak{g}}}(l, \bar{\Lambda})
$$

where $L_{\widehat{\mathfrak{g}}}(l, \bar{\Lambda})$ are level $l$ irreducible $\widehat{\mathfrak{g}}$-modules and $M_{\widehat{\mathfrak{g}}}(l, \bar{\Lambda})=\operatorname{Hom}_{\widehat{\mathfrak{g}}}\left(L_{\widehat{\mathfrak{g}}}(l, \bar{\Lambda}), L_{\widehat{\mathfrak{g}}}(1,0)^{\otimes l}\right)$ are vector spaces. Determining $M_{\widehat{\mathfrak{g}}}(l, \bar{\Lambda})$ is one of the main problems in representation theory which is amount to decomposing the tensor product. Unlike the question for finite dimensional Lie algebras (or corresponding Lie groups, quantum groups, etc), the vector spaces $M_{\widehat{\mathfrak{g}}}(l, \bar{\Lambda})$ are infinite dimensional. The obvious highest weight vector $v^{+} \otimes \cdots \otimes v^{+}$in $L_{\widehat{\mathfrak{g}}}(1,0)^{\otimes l}$ generates an irreducible $\widehat{\mathfrak{g}}$-submodule isomorphic to $L_{\widehat{\mathfrak{g}}}(l, 0)$. Thus $M_{\widehat{\mathfrak{g}}}(l, 0) \neq 0$. Since $L_{\widehat{\mathfrak{g}}}(1,0)$ and $L_{\widehat{\mathfrak{g}}}(l, 0)$ are vertex operator algebras, $L_{\widehat{\mathfrak{g}}}(1,0)^{\otimes l}$ has a tensor product vertex operator algebra structure with $L_{\widehat{\mathfrak{g}}}(l, 0)$ being a vertex operator subalgebra (with

2010 Mathematics Subject Classification. 17B69.

Jiang is supported by China NSF grants 10931006, 11371245, China RFDP grant 2010007310052, and the Innovation Program of Shanghai Municipal Education Commission (11ZZ18). 
a different conformal vector). In general, for a vertex operator algebra $V$ and a vertex operator subalgebra $U$ (with possibly different conformal vectors) of $V$, we denote by $C_{V}(U)$ the commutant of $U$ in $V$ (see [LL, 3.11] and $C_{V}(U)$ is a vertex operator subalgebra of $V$ (with possibly different conformal vectors). Then $M_{\widehat{\mathfrak{g}}}(l, 0)=C_{L_{\widehat{\mathfrak{g}}}(1,0) \otimes l}\left(L_{\widehat{\mathfrak{g}}}(l, 0)\right)$ is the commutant subalgebra of $L_{\widehat{\mathfrak{g}}}(l, 0)$ in $L_{\widehat{\mathfrak{g}}}(1,0)^{\otimes l}$ and is a simple vertex operator subalgebra of $L_{\widehat{\mathfrak{g}}}(1,0)^{\otimes l}$ and $M_{\widehat{\mathfrak{g}}}(l, \bar{\Lambda})$ are $M_{\widehat{\mathfrak{g}}}(l, 0)$-modules. In this paper, we first prove

Theorem 1.1. Let $L_{\widehat{\mathfrak{h}}}(n, 0)$ be the vertex operator subalgebra of $L_{\widehat{\mathfrak{s} \mathfrak{l}_{l}}}(n, 0)$ generated by the Cartan subalgebra h of $\mathfrak{s l}_{l}$. Then $C_{L_{\widehat{\mathfrak{s l n}}}(1,0) \otimes l}\left(L_{\widehat{\mathfrak{s l}_{n}}}(l, 0)\right) \cong C_{L_{\widehat{\mathfrak{s l l}}}(n, 0)}\left(L_{\widehat{\mathfrak{h}}}(n, 0)\right)$ as vertex operator algebras.

Note that $L_{\widehat{\mathfrak{s l}_{l}}}(n, 0)$ is an $\widehat{\mathfrak{s l}}_{l}$-module of level $n$ while $L_{\widehat{\mathfrak{s l}}_{n}}(l, 0)$ is an $\widehat{\mathfrak{s l}}_{n}$-module of level $l$. This provides another level-rank duality. The commutant $C_{L_{\widehat{\mathfrak{s}_{l}}}(n, 0)}\left(L_{\widehat{\mathfrak{h}}}(n, 0)\right)$ is the parafermion vertex operator algebra by physcists and denoted by $K\left(\mathfrak{s l}_{l}, n\right)$ which have been extensively studied [ZF], [CGT], [DLY2], [DLWY], [ALY], [DW1]-[DW3]. However, $C_{L_{\widehat{s} l_{l}}(n, 0)}\left(K\left(\mathfrak{s l}_{l}, n\right)\right)$ only contains $L_{\widehat{\mathfrak{h}}}(n, 0)$ and is a conformal extension of $L_{\widehat{\mathfrak{h}}}(n, 0)$ in $L_{\widehat{\mathfrak{s l}_{l}}}(n, 0)$. The proof of Theorem 1.1 also shows that

$$
C_{L_{\widehat{\mathfrak{I}_{l}}}(n, 0)}\left(K\left(\mathfrak{s} \mathfrak{l}_{l}, n\right)\right) \cong V_{\sqrt{n}} A_{l-1}
$$

which is a lattice vertex operator algebra corresponding to the lattice $\sqrt{n} A_{l-1}$. This follows from (3.5). Therefore $\left(K\left(\mathfrak{s l}_{l}, n\right), V_{\sqrt{n} A_{l-1}}\right)$ is a duality pair in the vertex operator algebra $L_{\widehat{\mathfrak{s l}_{l}}}(n, 0)$ in the following sense of [Ho2].

1.2. More generally, given a sequence of positive integers $\underline{\ell}=\left(l_{1}, \cdots l_{s}\right)$, the tensor product vertex operator algebra $L_{\widehat{\mathfrak{g}}}(\underline{\ell}, 0)=L_{\widehat{\mathfrak{g}}}\left(l_{1}, 0\right) \otimes L_{\widehat{\mathfrak{g}}}\left(l_{2}, 0\right) \otimes \cdots \otimes L_{\widehat{\mathfrak{g}}}\left(l_{s}, 0\right)$ contains a vertex operator subalgebra isomorphic to $L_{\widehat{\mathfrak{g}}}(|\underline{\ell}|, 0)$ with $|\underline{\ell}|=l_{1}+\cdots+l_{s}$. One considers the $\widehat{\mathfrak{g}}$-module decomposition:

$$
L_{\widehat{\mathfrak{g}}}(\underline{\ell}, 0)=\bigoplus L_{\widehat{\mathfrak{g}}}(|\underline{\ell}|, \bar{\Lambda}) \otimes M_{\widehat{\mathfrak{g}}}(\underline{\ell}, \bar{\Lambda})
$$

Then $M_{\widehat{\mathfrak{g}}}(\underline{\ell}, 0)=C_{L_{\widehat{\mathfrak{g}}}(\underline{\ell}, 0)}\left(L_{\widehat{\mathfrak{g}}}(|\underline{\ell}|, 0)\right)$ is a simple vertex operator algebra and all $M_{\widehat{\mathfrak{g}}}(\underline{\ell}, \bar{\Lambda})$ are $M_{\widehat{\mathfrak{g}}}(\underline{\ell}, 0)$-modules. On the other hand, the sequence $\underline{\ell}$ defines a Levi subalgebra $\mathfrak{l}_{\underline{\ell}}$ of $\mathfrak{s l}|\underline{\underline{\ell}}|$. The vertex operator subalgebra of $L_{\widehat{s \mid} \widehat{\mid \underline{\ell}}}(n, 0)$ generated by $\mathfrak{l}_{\underline{\ell}}$ is simple (but not rational) and is denoted by $L_{\widehat{\mathfrak{l}}_{\underline{\ell}}}(n, 0)$. Set $K\left(\mathfrak{s l}_{|\underline{\ell}|}, \mathfrak{l}_{\underline{\ell}}, n\right)=C_{L_{\left.\widehat{s}\right|_{\underline{\ell} \mid}}(n, 0)}\left(L_{\widehat{\mathfrak{l}}_{\underline{\ell}}}(n, 0)\right)$. This commutant subalgebra was first mentioned in [FZ]. Note that $\underline{l}_{\underline{\ell}}=\mathfrak{h}$ when $\underline{\ell}=(1, \cdots, 1)$ and $K\left(\mathfrak{s l}_{\underline{\ell} \underline{\ell}}, \mathfrak{l}_{\underline{\ell}}, n\right)=K\left(\mathfrak{s l}_{|\underline{\ell}|}, n\right)$ is the parafermion. With this setting, a more general rank-level duality holds.

Theorem 1.2. If $\mathfrak{g}=\mathfrak{s l}_{n}$, then $C_{L_{\widehat{\mathfrak{s l n}}}(\underline{\ell}, 0)}\left(L_{\widehat{\mathfrak{s l} n}}(|\underline{\ell}|, 0)\right) \cong C_{L_{\widehat{s}|\underline{\ell}|} \mid(n, 0)}\left(L_{\widehat{\widehat{l}}_{\underline{\underline{\ell}}}}(n, 0)\right)$ as vertex operator algebras. 
Similarly, the proof (cf. (4.2) ) of Theorem 1.2 also shows that $C_{L_{\widehat{s} \widehat{I}_{\underline{\ell}} \mid}(n, 0)}\left(K\left(\mathfrak{s l}_{|\underline{\ell}|}, \mathfrak{l}_{\underline{\ell}}, n\right)\right)$ is isomorphic to the tensor product of the vertex operator algebra corresponding to a semisimple Lie algebra and a lattice vertex operator algebra, and in particular is a rational vertex operator algebra.

1.3. Decomposing tensor products of representations has a long history in various representation theories such as finite groups, Lie groups, algebraic groups, quantum groups (and super-version) and many others to come. It is related to invariant theory, combinatorics, and many other fields. For example, the classical problem of decomposing the tensor power of the natural representation of classical Lie algebras (Lie groups, algebraic groups) has been in the heart of representation theory for over a century and it is still the driving motivation today for their quantum version and super-version. Although no single reference would do the justice for subject, we do refer to Weyl's book [Weyl] and Howe's lecture [Ho1], for much earlier work and its influence in mathematics, and to [LZ] for most recent results. In those cases, the component corresponding to one-dimensional trivial module is of the main interests and is the invariant subspace. However, for the affine Lie algebra $\widehat{\mathfrak{g}}$, the trivial module does not appear in the non-critical level, and the module $L_{\widehat{\mathfrak{g}}}(l, 0)$ plays the role of the identity object in the fusion tensor category and thus, the commutant $C_{L_{\widehat{\mathfrak{g}}}(1,0) \otimes l}\left(L_{\widehat{\mathfrak{g}}}(l, 0)\right)$ can be regarded as the invariant, which is a vertex operator algebra. Describing the commutant algebras in the algebras of operators on tensor powers is a main goal in invariant theory (see [LZ] for recent results). They are all related to Hecke algebras, Brauer algebras etc. In the classical cases, the commutant algebra is the invariant subalgebra of the endomorphism algebra of the tensor vector space.

In terms of tensor category $\mathcal{C}$ of representations, the commutant algebra is

$$
\operatorname{End}_{\mathcal{C}}\left(V^{\otimes l}\right)=\operatorname{Hom}_{\mathcal{C}}\left(\mathbb{I},\left(V^{\otimes l}\right)^{\vee} \otimes V^{\otimes l}\right)
$$

with $\mathbb{I}$ being the identity object of the tensor category $\mathcal{C}$. However, in case of vertex operator algebras, the commutant is

$$
C_{L_{\widehat{\mathfrak{g}}}(1,0) \otimes l}\left(L_{\widehat{\mathfrak{g}}}(l, 0)\right)=\operatorname{Hom}_{L_{\widehat{\mathfrak{g}}}(l, 0)}\left(L_{\widehat{\mathfrak{g}}}(l, 0), L_{\widehat{\mathfrak{g}}}(1,0)^{\otimes l}\right),
$$

where $\left.L_{\widehat{\mathfrak{g}}}(1,0)^{\otimes l}\right)$ is a vertex operator algebra while the tensor product is taken in the tensor category of representations of the affine Lie algebra $\widehat{\mathfrak{g}}$, rather than in the fusion tensor category of the vertex operator algebra. However $L_{\mathfrak{g}}(l, 0)$ is the identity object of the fusion tensor category of the vertex operator algebra $L_{\widehat{\mathfrak{g}}}(l, 0)$ and all other coefficients

$$
M_{\widehat{\mathfrak{g}}}(l, \bar{\Lambda})=\operatorname{Hom}_{L_{\widehat{\mathfrak{g}}}(l, 0)}\left(L_{\widehat{\mathfrak{g}}}(l, \bar{\Lambda}), L_{\widehat{\mathfrak{g}}}(1,0)^{\otimes l}\right)
$$

are $C_{L_{\widehat{\mathfrak{g}}}(1,0) \otimes l}\left(L_{\widehat{\mathfrak{g}}}(l, 0)\right)$-modules. From this aspect, the commutant vertex operator algebra $C_{L_{\widehat{\mathfrak{g}}}(1,0) \otimes l}\left(L_{\widehat{\mathfrak{g}}}(l, 0)\right)$ still plays the role of the endomorphism algebras as in the classical cases mentioned above. Thus describing the commutant algebras in tensor powers seems to be an invariant theory in the context of vertex operator algebras. 
It is well known that commutant construction of vertex operator algebra is the socalled coset construction in conformal field theory. The main question of computing the commutant subalgebras ware first raised in [FZ].

1.4. The classical Schur-Weyl duality builds the relation of the decomposition of the tensor power $V^{\otimes l}$ with the representations of the endomorphism ring. This relation builds connection between quite different representation theories, such as representations of general linear groups (algebraic, quantum, or their super version) with combinatorics (and geometry). Through this relations many properties of the representation theory of one side can be described by the other side. See [CLW] for recent application of this duality in characterization of the irreducible characters for certain Lie super algebras. In cases of representations of affine Lie algebras, Frenkel $[\mathrm{Fr}]$ found a duality between different affine Lie algebras of type $A$ at different levels. This duality now called level-rank duality, establishes a category equivalence between the category $\mathcal{C}\left(\widehat{\mathfrak{s l}}_{n}, l\right)$ of representations of $\widehat{\mathfrak{s l}}_{n}$ in level $l$ and the category $\mathcal{C}\left(\widehat{\mathfrak{s l}}_{l}, n\right)$. This equivalence has been made as equivalence of tensor categories recently in [OS] and its appearances have been found in many fields of mathematics, such as geometry and number theory, quantum groups, in addition to conformal field theory. See [ABS, NS, AS, Mu, MO]. Both Theorems 1.1 and 1.2 give another form of the level-rank duality. In particular, Theorem 1.2 can be regarded as a combination of level-rank duality and Howe duality. Further relations with those dualities still need to be investigated, in particular its interpretation in the geometric setting in terms of stable vector bundles over curves as in [MO].

1.5. Given a semi-simple Lie algebra $\mathfrak{g}$ and a levi subalgebra $\mathfrak{l}$, branching rule is to decompose irreducible $\mathfrak{g}$-modules into direct sums of irreducible $\mathfrak{l}$-modules. Similarly, one can consider the affine version of decomposing irreducible $\widehat{\mathfrak{g}}$-modules into direct sums of irreducible $\hat{\mathfrak{l}}$-modules, in the form of

$$
L_{\widehat{\mathfrak{g}}}(l, \bar{\Lambda})=\oplus L_{\hat{\mathfrak{l}}}\left(l, \bar{\Lambda}_{\mathfrak{l}}\right) \otimes N_{\mathfrak{g} \mathfrak{l l}}\left(l, \bar{\Lambda}, \bar{\Lambda}_{\mathfrak{l}}\right)
$$

In this case, $C_{L_{\widehat{\mathfrak{g}}}(l, 0)}\left(L_{\hat{\mathfrak{l}}}(l, 0)\right)=N_{\mathfrak{g}, \mathrm{l}}(l, 0,0)$ is a vertex operator algebra and all others $N_{\mathfrak{g}, \mathfrak{l}}\left(l, \bar{\Lambda}, \bar{\Lambda}_{\mathfrak{l}}\right)$ are the modules for this vertex operator algebra. When $\mathfrak{l}=\mathfrak{h}$ is the Cartan subalgebra of $\mathfrak{g}, C_{L_{\widehat{\mathfrak{g}}}(l, 0)}\left(L_{\hat{\mathfrak{l}}}(l, 0)\right)$ is called the parafermion, which has been studied extensively [ZF], [CGT], [DLY2], [DLWY], [ALY], [DW1]-[DW3], etc. For general Levisubalgebra $\mathfrak{l}$, we will call $C_{L_{\mathfrak{\mathfrak { g }}}(l, 0)}\left(L_{\mathfrak{l}}(l, 0)\right)$ the relative parafermion. Theorem 1.2 says that decomposition of tensor products of $\widehat{\mathfrak{s l}}_{n}$-irreducible integral representations at various levels $\left(l_{1}, \cdots, l_{s}\right)$ is related to the branching role of irreducible representations of $\widehat{\mathfrak{s l}}_{l}$ at the level $n$ with respect to the standard Levi subalgebra defined by $\left(l_{1}, \cdots, l_{s}\right)$. In $[\mathrm{BEHHH}]$, it was suggested that certain unifying $\mathcal{W}$-algebras can be realized in terms of coset construction via embedding $\mathfrak{s l}_{n}$ into $\mathfrak{s l}_{n+1}$. Theorem 1.2 is a more general form of this suggestion. 
In fact, the left hand side corresponds to the conformal field theory coset construction $\mathfrak{s u}(n)_{l_{1}} \oplus \cdots \oplus \mathfrak{s u}(n)_{l_{s}} / \mathfrak{s u}(n)_{l_{1}+\cdots+l_{s}}$ while the right hand side corresponds to the conformal field theory coset construction $\mathfrak{s u}\left(l_{1}+\cdots+l_{s}\right)_{n} / \mathfrak{l}\left(l_{1}, \cdots, l_{s}\right)_{n}$. Theorem 1.2 seems to resemble a version of Howe reciprocity property of branching rules [HTW]. It seems the pairs satisfy the Gelfand pair (or Howe's symmetric pair) properties in the context of vertex operator algebras.

In [Ho1], Howe studied a pair of reductive subgroups $\left(G_{1}, G_{2}\right)$ in a given reductive Lie group $G$ such that $G_{1}$ and $G_{2}$ are mutually centralizers and a certain representation $\pi$ of $G$ has a tensor decomposition into direct sum of the form

$$
\pi=\sum_{\tau} \tau \otimes \rho(\tau)
$$

such that $\rho: \operatorname{Irr}\left(G_{1}\right) \rightarrow \operatorname{Irr}\left(G_{2}\right)$ defines a correspondence. In terms of vertex operator algebras, Theorem 1.1 implies that $L_{\mathfrak{s l n}_{n}}(l, 0)$ and $K\left(\mathfrak{s l}_{l}, n\right)$ form a duality pair in $V_{A_{n-1}^{\times l}}$ while $K(\mathfrak{s l} l, n)$ and $V_{\sqrt{n} A_{l-1}}$ form a duality pair in $L_{\widehat{\mathfrak{s} l}}(n, 0)$. More details of correspondences of irreducible modules will be discussed in Section 5. Theorem 1.2 provides two other duality pairs. These pairs satisfy certain reciprocity law of duality pairs as discussed in [Ho2].

1.6. Various decompositions of the tensor product $L_{\widehat{\mathfrak{g}}}(1,0)^{\otimes l}$ have been studied extensively. The commutant vertex operator algebra $C=C_{L_{\widehat{\mathfrak{g}}}(1,0) \otimes l}\left(L_{\widehat{\mathfrak{g}}}(l, 0)\right)$ is called coset construction. They are expected to be rational. In case of $\mathfrak{g}=\mathfrak{s l}_{2}$, we proved the rationality for all $l$ in [JL]. For smaller $l=2,3$ there has been a lot of computational results. In [JL] all irreducible modules for the commutant algebra have been classified. Not all irreducible $C$-modules appear as $M_{\widehat{\mathfrak{g}}}(l, \bar{\Lambda})$ in the decomposition (1.1), but they appear in the decompositions of $\otimes_{i=1}^{l} L_{\widehat{\mathfrak{g}}}\left(1, \bar{\Lambda}_{i}\right)$. This is quite different from the classical Schur duality for associative algebras for which all irreducible modules of the commutant algebra appear in the decomposition. For $\mathfrak{g}=\mathfrak{s l}_{n}$ with higher rank, rationality question is still illusive. On the other hand, the parafermion vertex operator algebras have been studied extensively recently and have been expected to be rational as well [DLY2], [DLWY], [ALY], [DW1]-[DW3]. Theorem 1.1 explains that cost-constructions and parafermions are the same. Establishment of rationality of one would give the other. Theorem 1.1 might provide a way to toward answering the rationality question.

The idea of establishing Theorems 1.1 and 1.2 is to use the fact that $L_{\widehat{\mathfrak{g}}}(1,0)$ is a lattice vertex operator algebra corresponding to the root lattice $A_{n-1}$. Thus the tensor product in (1.1) is the lattice vertex operator algebra corresponding to the lattice $A_{n-1}^{\times l}$ which contains a sublattice $N_{n}^{l}$ such that the commutant of the Heisenberg vertex operator algebra $L_{\widehat{\mathfrak{h}}}(l, 0)$ in $L_{\mathfrak{\mathfrak { g }}}(1,0)^{\otimes l}$ is isomorphic to the lattice vertex operator algebra $V_{N_{n}^{l}}$ which particularly contains $C_{L_{\widehat{\mathfrak{g}}}(1,0) \otimes l}\left(L_{\widehat{\mathfrak{g}}}(l, 0)\right)$. On the other hand, the commutant of $C_{L_{\widehat{\mathfrak{g}}}(1,0) \otimes l}\left(L_{\widehat{\mathfrak{g}}}(l, 0)\right)$ in $V_{N_{n}^{l}}$ is the parafermion $K\left(\mathfrak{s l}_{n}, l\right)$ (3.13). A similarly defined lattice 
$\tilde{N}_{l}^{n} \subseteq \widetilde{A}_{l-1}^{\times n}$ such that $V_{\widetilde{N}_{n}^{l}}$ contains the parafermion $K\left(\mathfrak{s}_{l}, n\right)$. Although the two lattices $N_{n}^{l}$ and $\widetilde{N}_{l}^{n}$ are isomorphic, the isomorphism between $V_{N_{n}^{l}}$ and $V_{\widetilde{N}_{l}^{n}}$ we needed is not the one coming from this lattice isomorphism. A key point is to find a correct isomorphism from $V_{N_{n}^{l}}$ to $V_{\widetilde{N}_{l}^{n}}$, so that $C_{L_{\widehat{\mathfrak{g}}}(1,0) \otimes l}\left(L_{\widehat{\mathfrak{g}}}(l, 0)\right)$ is mapped to $K\left(\mathfrak{s} \mathfrak{l}_{l}, n\right)$ in $V_{\widetilde{N}_{l}^{n}}$.

1.7. We now outline the paper. In Section 2, we briefly review basics on vertex operator algebras which we need in the latter sections. In Section 3, we establish Theorem 1.1. Theorem 1.2 is proved in Section 4. In Section 5, we discuss various duality pairs and reciprocity laws in the spirit of [Ho1] and [Ho2] that appear in the context of vertex operator algebras appeared in this paper. Such properties called SeeSaw property in [Ku] in the context of reductive groups. In Section 6 we summarize results in answering the rationality of parafermion vertex operator algebras and coset constructions.

Acknowledgement: This work is a continuation of the effort to understand the decomposition of the tensor product of basic representation in the spirit of Schur-Weyl duality. This work started when the second author was visiting SJTU during the summer of 2013. The second author thanks the support by SJTU and its hospitality. The main results of the paper were achieved during the visit of the first author to Kansas State University in January 2014. This visit was supported by a collaborative project between the departments of Mathematics of SJTU and Kansas State University funded by SJTU. The first author thanks C. Lam for informing her, after her talk on this paper at a conference in Dalian, June 14-17, 2014, that he also has proved a result similar to Theorem 1.1.

\section{Preliminaries}

Let $V=(V, Y, \mathbf{1}, \omega)$ be a vertex operator algebra [B], [FLM], [LL]. We review various notions of $V$-modules and the definition of rational vertex operator algebras and some basic facts (cf. [FLM], [Z], [DLM3], [DLM4], [LL]). We also recall intertwining operators, fusion rules and some consequences following $[\mathrm{FHL}],[\mathrm{ADL}],[\mathrm{W}],[\mathrm{DMZ}],[\mathrm{A}],[\mathrm{DJL}]$.

Definition 2.1. A weak $V$-module is a vector space $M$ equipped with a linear map

$$
\begin{aligned}
Y_{M}: & V \rightarrow \operatorname{End}(M)\left[\left[z, z^{-1}\right]\right] \\
& v \mapsto Y_{M}(v, z)=\sum_{n \in \mathbb{Z}} v_{n} z^{-n-1}, v_{n} \in \operatorname{End}(M)
\end{aligned}
$$

satisfying the following:

1) $v_{n} w=0$ for $n>>0$ where $v \in V$ and $w \in M$,

2) $Y_{M}(\mathbf{1}, z)=\operatorname{id}_{M}$, 
3) The Jacobi identity holds:

$$
\begin{gathered}
z_{0}^{-1} \delta\left(\frac{z_{1}-z_{2}}{z_{0}}\right) Y_{M}\left(u, z_{1}\right) Y_{M}\left(v, z_{2}\right)-z_{0}^{-1} \delta\left(\frac{z_{2}-z_{1}}{-z_{0}}\right) Y_{M}\left(v, z_{2}\right) Y_{M}\left(u, z_{1}\right) \\
=z_{2}^{-1} \delta\left(\frac{z_{1}-z_{0}}{z_{2}}\right) Y_{M}\left(Y\left(u, z_{0}\right) v, z_{2}\right) .
\end{gathered}
$$

Definition 2.2. An admissible $V$ module is a weak $V$ module which carries a $\mathbb{Z}_{+- \text {grading }}$ $M=\bigoplus_{n \in \mathbb{Z}_{+}} M(n)$, such that if $v \in V_{r}$ then $v_{m} M(n) \subseteq M(n+r-m-1)$.

Definition 2.3. An ordinary $V$ module is a weak $V$ module which carries a $\mathbb{C}$-grading $M=\bigoplus_{\lambda \in \mathbb{C}} M_{\lambda}$, such that:

1) $\operatorname{dim}\left(M_{\lambda}\right)<\infty$,

2) $M_{\lambda+n}=0$ for fixed $\lambda$ and $n<<0$,

3) $L(0) w=\lambda w=\operatorname{wt}(w) w$ for $w \in M_{\lambda}$ where $L(0)$ is the component operator of $Y_{M}(\omega, z)=\sum_{n \in \mathbb{Z}} L(n) z^{-n-2}$.

Remark 2.4. It is easy to see that an ordinary $V$-module is an admissible one. If $W$ is an ordinary $V$-module, we simply call $W$ a $V$-module.

We call a vertex operator algebra rational if the admissible module category is semisimple. We have the following result from [DLM3] (also see [Z]).

Theorem 2.5. If $V$ is a rational vertex operator algebra, then $V$ has finitely many irreducible admissible modules up to isomorphism and every irreducible admissible $V$-module is ordinary.

Suppose that $V$ is a rational vertex operator algebra and let $M^{1}, \ldots, M^{k}$ be the irreducible modules such that

$$
M^{i}=\oplus_{n \geq 0} M_{\lambda_{i}+n}^{i}
$$

where $\lambda_{i} \in \mathbb{Q}$ [DLM4], $M_{\lambda_{i}}^{i} \neq 0$ and each $M_{\lambda_{i}+n}^{i}$ is finite dimensional.

A vertex operator algebra is called $C_{2}$-cofinite if $C_{2}(V)$ has finite codimension where $C_{2}(V)=\left\langle u_{-2} v \mid u, v \in V\right\rangle[\mathrm{Z}]$.

Remark 2.6. If $V$ is a vertex operator algebra satisfying $C_{2}$-cofinite property, $V$ has only finitely many irreducible admissible modules up to isomorphism [DLM3], [L], [Z].

We now recall lattice vertex operator algebras and related results. Let $L$ be a positive definite even lattice in the sense that $L$ has a symmetric positive definite $\mathbb{Z}$-valued bilinear form $(\cdot, \cdot)$ such that $(\alpha, \alpha) \in 2 \mathbb{Z}$ for any $\alpha \in L$. We set $\mathfrak{h}=\mathbb{C} \otimes_{\mathbb{Z}} L$ and extend $(\cdot, \cdot)$ to a $\mathbb{C}$-bilinear form on $\mathfrak{h}$. Let $\hat{\mathfrak{h}}=\mathbb{C}\left[t, t^{-1}\right] \otimes \mathfrak{h} \oplus \mathbb{C} C$ be the affinization of commutative Lie algebra $\mathfrak{h}$ defined by

$$
\left[\beta_{1} \otimes t^{m}, \beta_{2} \otimes t^{n}\right]=m\left(\beta_{1}, \beta_{2}\right) \delta_{m,-n} C \text { and }[C, \hat{\mathfrak{h}}]=0
$$


for any $\beta_{i} \in \mathfrak{h}, m, n \in \mathbb{Z}$. Then $\hat{\mathfrak{h}}^{\geq 0}=\mathbb{C}[t] \otimes \mathfrak{h} \oplus \mathbb{C} C$ is a commutative subalgebra. For any $\lambda \in \mathfrak{h}$, we can define a one dimensional $\hat{\mathfrak{h}}^{\geq 0}$-module $\mathbb{C} e^{\lambda}$ by the actions $\rho\left(h \otimes t^{m}\right) e^{\lambda}=$ $(\lambda, h) \delta_{m, 0} e^{\lambda}$ and $\rho(C) e^{\lambda}=e^{\lambda}$ for $h \in \mathfrak{h}$ and $m \geq 0$. Now we denote by

$$
M(1, \lambda)=U(\hat{\mathfrak{h}}) \otimes_{U(\hat{\mathfrak{h}} \geq 0)} \mathbb{C} e^{\lambda} \cong S\left(t^{-1} \mathbb{C}\left[t^{-1}\right] \otimes \mathfrak{h}\right)
$$

the $\hat{\mathfrak{h}}$-module induced from $\hat{\mathfrak{h}}^{\geq 0}$-module $\mathbb{C} e^{\lambda}$. Set $M(1)=M(1,0)$. Then there exists a linear map $Y: M(1) \rightarrow\left(\operatorname{End} M(1, \lambda)\left[\left[z, z^{-1}\right]\right]\right.$ such that $(M(1), Y, \mathbf{1}, \omega)$ has a simple vertex operator algebra structure and $(M(1, \lambda), Y)$ becomes an irreducible $M(1)$-module for any $\lambda \in \mathfrak{h}$ (see $[\mathrm{FLM}]$ ). The vacuum vector and the Virasoro element are given by $\mathbf{1}=e^{0}$ and $\omega=\frac{1}{2} \sum_{a=1}^{d} h_{a}(-1)^{2} \mathbf{1}$ respectively, where $\left\{h_{a} \mid a=1,2, \cdots, d\right\}$ is an orthonormal basis of $\mathfrak{h}$.

Let $\widehat{L}$ be the canonical central extension of $L$ by $\langle\kappa\rangle=\left\langle\kappa \mid \kappa^{2}=1\right\rangle$ (see [FLM]):

$$
1 \rightarrow\langle\kappa\rangle \rightarrow \widehat{L} \rightarrow L \rightarrow 1
$$

with the commutator map $c(\alpha, \beta)=\kappa^{(\alpha, \beta)}$ for $\alpha, \beta \in L$. Let $e: L \rightarrow \widehat{L}\left(\alpha \mapsto e_{\alpha}\right)$ be a section such that $e(0)=1$ and $\epsilon: L \times L \rightarrow\langle\kappa\rangle$ the corresponding 2-cocycle. We may assume that $\epsilon$ is bimultiplicative. Then $\epsilon(\alpha, \beta) \epsilon(\beta, \alpha)=\kappa^{(\alpha, \beta)}$,

$$
\epsilon(\alpha, \beta) \epsilon(\alpha+\beta, \gamma)=\epsilon(\beta, \gamma)(\alpha, \beta+\gamma)
$$

and $e_{\alpha} e_{\beta}=\epsilon(\alpha, \beta) e_{\alpha+\beta}$ for $\alpha, \beta, \gamma \in L$. Let $\theta$ denote the automorphism of $\widehat{L}$ defined by $\theta\left(e_{\alpha}\right)=e_{-\alpha}$ and $\theta(\kappa)=\kappa$. Set $K=\left\{a^{-1} \theta(a) \mid a \in \widehat{L}\right\}$. Note that if $(\alpha, \beta) \in 2 \mathbb{Z}$ for all $\alpha, \beta \in L$ then $\widehat{L}=L \times\langle\kappa\rangle$ is a direct product of abelian groups. In this case we may choose $\epsilon(\alpha, \beta)=1$ for all $\alpha, \beta \in L$.

The lattice vertex operator algebra associated to $L$ is given by

$$
V_{L}=M(1) \otimes \mathbb{C}^{\epsilon}[L]
$$

where $\mathbb{C}^{\epsilon}[L]$ is the twisted group algebra of $L$ with a basis $e^{\alpha}$ for $\alpha \in L$ and is an $\widehat{L}$-module such that $e_{\alpha} e^{\beta}=\epsilon(\alpha, \beta) e^{\alpha+\beta}$. Note that if $(\alpha, \beta) \in 2 \mathbb{Z}$ for all $\alpha, \beta \in L$ then $\mathbb{C}^{\epsilon}[L]=\mathbb{C}[L]$ is the usual group algebra.

Recall that $L^{\circ}=\{\lambda \in \mathfrak{h} \mid(\alpha, \lambda) \in \mathbb{Z}\}$ is the dual lattice of $L$. There is an $\widehat{L}$-module structure on $\mathbb{C}\left[L^{\circ}\right]=\bigoplus_{\lambda \in L^{\circ}} \mathbb{C} e^{\lambda}$ such that $\kappa$ acts as -1 (see [DL]). Let $L^{\circ}=\cup_{i \in L^{\circ} / L}(L+$ $\left.\lambda_{i}\right)$ be the coset decomposition such that $\left(\lambda_{i}, \lambda_{i}\right)$ is minimal among all $(\lambda, \lambda)$ for $\lambda \in L+\lambda_{i}$. In particular, $\lambda_{0}=0$. Set $\mathbb{C}\left[L+\lambda_{i}\right]=\bigoplus_{\alpha \in L} \mathbb{C} e^{\alpha+\lambda_{i}}$. Then $\mathbb{C}\left[L^{\circ}\right]=\bigoplus_{i \in L^{\circ} / L} \mathbb{C}\left[L+\lambda_{i}\right]$ and each $\mathbb{C}\left[L+\lambda_{i}\right]$ is an $\widehat{L}$-submodule of $\mathbb{C}\left[L^{\circ}\right]$. The action of $\widehat{L}$ on $\mathbb{C}\left[L+\lambda_{i}\right]$ is as follows:

$$
e_{\alpha} e^{\beta+\lambda_{i}}=\epsilon(\alpha, \beta) e^{\alpha+\beta+\lambda_{i}}
$$

for $\alpha, \beta \in L$. Although it looks that the $\hat{L}$-module structure on each $\mathbb{C}\left[L+\lambda_{i}\right]$ depends on the choice of $\lambda_{i}$ in $L+\lambda_{i}$, it is easy to check that different choices of $\lambda_{i}$ give isomorphic $\widehat{L}$-modules. 
We now recall the notion of intertwining operators and fusion rules from [FHL].

Definition 2.7. Let $V$ be a vertex operator algebra and $M^{1}, M^{2}, M^{3}$ be weak $V$-modules. An intertwining operator $\mathcal{Y}(\cdot, z)$ of type $\left(\begin{array}{c}M^{3} \\ M^{1} M^{2}\end{array}\right)$ is a linear map

$$
\begin{gathered}
\mathcal{Y}(\cdot, z): M^{1} \rightarrow \operatorname{Hom}\left(M^{2}, M^{3}\right)\{z\} \\
v^{1} \mapsto \mathcal{Y}\left(v^{1}, z\right)=\sum_{n \in \mathbb{C}} v_{n}^{1} z^{-n-1}
\end{gathered}
$$

satisfying the following conditions:

(1) For any $v^{1} \in M^{1}, v^{2} \in M^{2}$ and $\lambda \in \mathbb{C}, v_{n+\lambda}^{1} v^{2}=0$ for $n \in \mathbb{Z}$ sufficiently large.

(2) For any $a \in V, v^{1} \in M^{1}$,

$$
\begin{gathered}
z_{0}^{-1} \delta\left(\frac{z_{1}-z_{2}}{z_{0}}\right) Y_{M^{3}}\left(a, z_{1}\right) \mathcal{Y}\left(v^{1}, z_{2}\right)-z_{0}^{-1} \delta\left(\frac{z_{1}-z_{2}}{-z_{0}}\right) \mathcal{Y}\left(v^{1}, z_{2}\right) Y_{M^{2}}\left(a, z_{1}\right) \\
=z_{2}^{-1} \delta\left(\frac{z_{1}-z_{0}}{z_{2}}\right) \mathcal{Y}\left(Y_{M^{1}}\left(a, z_{0}\right) v^{1}, z_{2}\right)
\end{gathered}
$$

(3) For $v^{1} \in M^{1}, \frac{d}{d z} \mathcal{Y}\left(v^{1}, z\right)=\mathcal{Y}\left(L(-1) v^{1}, z\right)$.

All of the intertwining operators of type $\left(\begin{array}{c}M^{3} \\ M^{1} M^{2}\end{array}\right)$ form a vector space denoted by $I_{V}\left(\begin{array}{c}M^{3} \\ M^{1} M^{2}\end{array}\right)$. The dimension of $I_{V}\left(\begin{array}{c}M^{3} \\ M^{1} M^{2}\end{array}\right)$ is called the fusion rule of type $\left(\begin{array}{c}M^{3} \\ M^{1} M^{2}\end{array}\right)$ for $V$.

We have the following results from $[\mathrm{D}]$ and $[\mathrm{DL}]$.

Theorem 2.8. Let $L$ be a positive-definite even lattice. Then

(1) $V_{L}$ is rational and $V_{L+\lambda_{i}}, i \in L^{\circ} / L$ are all the irreducible modules of $V_{L}$.

(2) For $\lambda, \mu, \gamma \in L^{\circ}$,

$$
I_{V_{L}}\left(\begin{array}{c}
V_{L+\gamma} \\
V_{L+\lambda} V_{L+\mu}
\end{array}\right) \neq 0
$$

if and only if $\gamma-\lambda-\mu \in L$.

\section{LeVEL-RANK DUALity}

For $k \in \mathbb{Z}_{+}$and a complex finite-dimensional simple Lie algebra $\mathfrak{g}$ with normalized non-degenerate bilinear form, let $\widehat{\mathfrak{g}}$ be the corresponding affine Lie algebra and $L_{\widehat{\mathfrak{g}}}(k, 0)$ the simple vertex operator algebra associated with the integrable highest weight module 
of $\widehat{\mathfrak{g}}$ with level $k$. Let $\mathfrak{h}$ be the Cartan subalgebra of $\mathfrak{g}$ and $L_{\widehat{\mathfrak{h}}}(k, 0)$ the Heisenberg vertex operator subalgebra of $L_{\widehat{\mathfrak{g}}}(k, 0)$ generated by $\mathfrak{h}$. Let

$$
K(\mathfrak{g}, k)=\left\{v \in L_{\widehat{\mathfrak{g}}}(k, 0) \mid\left[Y\left(u, z_{1}\right), Y\left(v, z_{2}\right)\right]=0, u \in L_{\widehat{\mathfrak{h}}}(k, 0)\right\} .
$$

Then $K\left(L_{\widehat{\mathfrak{g}}}(k, 0)\right)$ is the so-called parafermion vertex operator algebra (see [BEHHH], [DLY2], [DW2]).

Let $s \in \mathbb{Z}_{\geq 2}$ and $\underline{\ell}=\left(l_{1}, \cdots, l_{s}\right)$ such that $l_{1}, \cdots, l_{s} \in \mathbb{Z}_{+}$. Let $L_{\mathfrak{\mathfrak { g }}}\left(l_{i}, 0\right)$ be the simple vertex operator algebra associated with the integrable highest weight module of $\widehat{\mathfrak{g}}$ with level $l_{i}, i=1,2, \cdots, s$. Then we have the tensor product vertex operator algebra:

$$
V=L_{\widehat{\mathfrak{g}}}\left(l_{1}, 0\right) \otimes L_{\widehat{\mathfrak{g}}}\left(l_{2}, 0\right) \otimes \cdots \otimes L_{\widehat{\mathfrak{g}}}\left(l_{s}, 0\right) .
$$

Denote

$$
l=|\underline{\ell}|=\sum_{i=1}^{s} l_{i} .
$$

$\mathfrak{g}$ can be naturally imbedded into the weight one subspace of $V$ diagonally as follows:

$$
\begin{gathered}
\mathfrak{g} \hookrightarrow V_{1} \subseteq V \\
a \mapsto a(-1) \mathbf{1} \otimes \mathbf{1} \otimes \cdots \otimes \mathbf{1}+\mathbf{1} \otimes a(-1) \mathbf{1} \otimes \mathbf{1} \otimes \cdots \otimes \mathbf{1}+\mathbf{1} \otimes \cdots \otimes \mathbf{1} \otimes a(-1) \mathbf{1} .
\end{gathered}
$$

It is known that the vertex operator subalgebra $U$ of $V$ generated by $\mathfrak{g}$ is isomorphic to the simple vertex operator algebra $L_{\widehat{\mathfrak{g}}}(l, 0)$ (see for example [FZ] and [LL]). Set

$$
C_{V}(U)=\left\{v \in V \mid\left[Y\left(u, z_{1}\right), Y\left(v, z_{2}\right)\right]=0, u \in U\right\} .
$$

Then (see $[\mathrm{LL}])$

$$
C_{V}(U)=\left\{v \in V \mid u_{m} v=0, u \in U, m \geq 0\right\}
$$

is a vertex operator subalgebra of $V$ with a different conformal vector. We will denote $L_{\widehat{\mathfrak{g}}}(l, \bar{\Lambda})$ the irreducible module of the vertex operator algebra $L_{\widehat{\mathfrak{g}}}(l, 0)$ associated to the highest weight $\Lambda$ of $\widehat{\mathfrak{g}} . \bar{\Lambda}=\left.\Lambda\right|_{\mathfrak{h}}$ is defined as follows ( see $\S 6.2$ in $[\mathrm{K}]$ ):

$$
\Lambda=\bar{\Lambda}+l \Lambda_{0}+m \delta
$$

for some $m \in \mathbb{Z}$, where $\Lambda_{0}$ is the highest weight of $L_{\widehat{\mathfrak{g}}}(1,0)$ as an irreducible highest weight $\widehat{\mathfrak{g}}$-module. We have the following lemma.

Lemma 3.1. $C_{V}(U)$ is a simple vertex operator subalgebra of $V$.

Proof. Since $U$ is rational, it follows that as a $U$-module $V$ is completely reducible. Then

$$
V=U \otimes C_{V}(U) \bigoplus_{\Lambda} L_{\mathfrak{g}}(l, \bar{\Lambda}) \otimes M(\Lambda),
$$

with the summation over $\Lambda$ such that $\bar{\Lambda} \neq 0$ and $L_{\widehat{\mathfrak{g}}}(l, \bar{\Lambda})$ are irreducible $L_{\widehat{\mathfrak{g}}}(l, 0)$-modules and $M(\Lambda)$ are modules of $C_{V}(U)$. Let $W$ be a non-zero ideal of $C_{V}(U)$. Note that for any $u \in U \otimes W, v \in L_{\widehat{\mathfrak{g}}}(l, \bar{\Lambda}) \otimes M(\Lambda)$, and $m \in \mathbb{Z}$, we have $u_{m} v \in L_{\widehat{\mathfrak{g}}}(l, \bar{\Lambda}) \otimes M(\Lambda)$. By [LL, Cor. 4.5.10, Prop. 3.1.19], the intersection of $U \otimes C_{V}(U)$ and the ideal of $V$ containing $U \otimes W$ is $U \otimes W$. Since $V$ is simple, it follows that $W=C_{V}(U)$. 
In the rest of this paper, we always assume that $\mathfrak{g}=\mathfrak{s l}_{n}(\mathbb{C})(n \geq 2)$ is the simple Lie algebra of type $A_{n-1}$ over $\mathbb{C}$. In this section we study the case that $s \geq 2$ and $l_{1}=\cdots=l_{s}=1$. Then $l=|\underline{\ell}|=s$ and

$$
V=L_{\widehat{\mathfrak{s l n}}}(1,0)^{\otimes l}, \quad U \cong L_{\mathfrak{s l n}_{n}}(l, 0) .
$$

Note that $L_{\widehat{\mathfrak{s l}_{n}}}(1,0) \cong V_{A_{n-1}}$, where $V_{A_{n-1}}$ is the lattice vertex operator algebra associated to the root lattice $A_{n-1}$.

Let

$$
A_{n-1}^{\times l}=\bigoplus_{i=1}^{n-1} \bigoplus_{j=1}^{l} \mathbb{Z} \alpha^{i j}
$$

be a lattice such that for $1 \leq i, p \leq n-1,1 \leq j, q \leq l$,

$$
\begin{gathered}
\left(\alpha^{i j}, \alpha^{p q}\right)=0, \text { if } j \neq q, \\
\left(\alpha^{i j}, \alpha^{p j}\right)= \begin{cases}2, & \text { if } p=i, \\
-1, & \text { if } i=p-1 \text { or } i=p+1, \\
0 & \text { otherwise. }\end{cases}
\end{gathered}
$$

Then the lattice vertex operator algebra $V_{A_{n-1}^{\times l}}$ is isomorphic to $V$ and the $j^{\text {th }}$ factor of $V$ is the lattice vertex operator algebra $V_{A_{n-1}^{(j)}} \cong V_{A_{n-1}}$ with $A_{n-1}^{(j)}=\bigoplus_{i=1}^{n-1} \mathbb{Z} \alpha^{i j}$.

For $1 \leq i \leq n-1$, define

$$
e^{i}=\sum_{j=1}^{l} e^{\alpha^{i j}}, e^{-i}=\sum_{j=1}^{l} e^{-\alpha^{i j}} .
$$

Since $L_{\widehat{\mathfrak{g}}}(l, 0)$ is generated by $e^{i}, e^{-i}, i=1,2, \cdots, n-1$, it follows that

$$
C_{V}(U)=\left\{v \in V \mid e_{m}^{i} v=e_{m}^{-i} v=0,1 \leq i \leq n-1, m \geq 0\right\} .
$$

Let $N_{n}^{l}$ be the sublattice

$$
N_{n}^{l}=\bigoplus_{i=1}^{n-1} \bigoplus_{j=1}^{l-1} \mathbb{Z}\left(\alpha^{i j}-\alpha^{i, j+1}\right) \subseteq A_{n-1}^{\times l}
$$

(with the restriction of the bilinear form of $A_{n-1}^{\times l}$ ) and $V_{N_{n}^{l}}$ the vertex operator algebra associated with $N_{n}^{l}$. It is obvious that $V_{N_{n}^{l}} \subseteq V$. Set

$$
K=\bigoplus_{i=1}^{n-1} \mathbb{Z} \alpha^{i}, \mathfrak{h}_{n}=\bigoplus_{i=1}^{n-1} \mathbb{C} \alpha^{i}(-1) \mathbf{1}
$$

where $\alpha^{i}=\sum_{j=1}^{l} \alpha^{i j}, \alpha^{i}(-1) \mathbf{1}=\sum_{j=1}^{l} \alpha^{i j}(-1) \mathbf{1}, i=1,2, \cdots, n-1, j=1,2, \cdots, l$. Then $K \cong \sqrt{l} A_{n-1}$. 
Let $L_{\widehat{\mathfrak{h}_{n}}}(l, 0) \subseteq L_{\widehat{\mathfrak{s l n}_{n}}}(l, 0)$ be a Heisenberg vertex operator subalgebra of $V$ generated by $\mathfrak{h}_{n}$. Then as an $L_{\widehat{\mathfrak{h}}_{n}}(l, 0)$-module, $V$ is completely reducible. Note that $V$ is linearly spanned by

$$
\gamma^{1}\left(-m_{1}\right) \cdots \gamma^{k}\left(-m_{k}\right) \otimes e^{\alpha}, \gamma^{1}, \cdots, \gamma^{k}, \alpha \in A_{n-1}^{\times l}, k \geq 0, m_{1} \geq m_{2} \geq \cdots \geq m_{k} \geq 1
$$

and $\left(\sum_{j=1}^{l} \alpha^{i j}, \alpha^{p q}-\alpha^{p, q+1}\right)=0$ for any $1 \leq i, p \leq n-1,1 \leq q \leq l$. Note also that

$$
\mathbb{C} \otimes_{\mathbb{Z}} A_{n-1}^{\times l}=\mathbb{C} \otimes_{\mathbb{Z}} K \oplus \mathbb{C} \otimes_{\mathbb{Z}} N_{n}^{l} .
$$

Then it is easy to see that as an $\widehat{\mathfrak{h}}_{n}$-module, $V$ is a direct sum of highest weight modules of $\widehat{\mathfrak{h}}_{n}$ with highest weight vectors

$$
h^{1}\left(-m_{1}\right) \cdots h^{k}\left(-m_{k}\right) \otimes e^{\alpha}, h^{1}, \cdots, h^{k} \in N_{n}^{l}, \alpha \in A_{n-1}^{\times l}, k \geq 0, m_{1} \geq m_{2} \geq \cdots \geq m_{k} \geq 1 .
$$

Then we have

$$
V_{N_{n}^{l}}=\left\{v \in V \mid \sum_{j=1}^{l} \alpha^{i j}(m) v=0,1 \leq i \leq n-1, m \geq 0\right\} .
$$

So we get the following lemma.

Lemma 3.2. $C_{V}\left(L_{\widehat{\mathfrak{h}_{n}}}(l, 0)\right)=V_{N_{n}^{l}}$. In particular, $C_{V}(U) \subseteq V_{N_{n}^{l}}$.

For $1 \leq i \leq n-1$, we have

$$
e_{-1}^{i} e^{-i}=\frac{1}{2} \sum_{j=1}^{l} \alpha^{i j}(-2) \mathbf{1}+\frac{1}{2} \sum_{j=1}^{l} \alpha^{i j}(-1)^{2} \mathbf{1}+\sum_{1 \leq p<q \leq l}\left(e^{\alpha^{i p}-\alpha^{i q}}+e^{-\alpha^{i p}+\alpha^{i q}}\right) .
$$

Note that

$$
\sum_{j=1}^{l} \alpha^{i j}(-1)^{2} \mathbf{1}=\frac{1}{l} \sum_{1 \leq p<q \leq l}\left(\alpha^{i p}-\alpha^{i q}\right)(-1)^{2} \mathbf{1}+\frac{1}{l}\left(\alpha^{i 1}+\cdots+\alpha^{i l}\right)(-1)^{2} \mathbf{1} .
$$

So on $V_{N_{n}^{l}}$ we have

$$
\left(e_{-1}^{i} e^{-i}\right)_{m}=\sum_{1 \leq p<q \leq l}\left[\frac{1}{2 l}\left(\alpha^{i p}-\alpha^{i q}\right)(-1)^{2} \mathbf{1}+\left(e^{\alpha^{i p}-\alpha^{i q}}+e^{-\alpha^{i p}+\alpha^{i q}}\right)\right]_{m}, m \geq 0 .
$$

For $1 \leq i \leq n-1$, set

$$
{ }^{\prime} \omega^{i}=\frac{1}{l+2} \sum_{1 \leq p<q \leq l}\left[\frac{1}{2 l}\left(\alpha^{i p}-\alpha^{i q}\right)(-1)^{2} \mathbf{1}+\left(e^{\alpha^{i p}-\alpha^{i q}}+e^{-\alpha^{i p}+\alpha^{i q}}\right)\right] .
$$

The following lemma can be checked directly.

Lemma 3.3. For $1 \leq i \leq n-1,{ }^{\prime} \omega^{i}$ is a Virasoro vector of $V$ with central charge $\frac{2(l-1)}{l+2}$. 
Let $K$ be defined as in (3.1) and $V_{K}$ the lattice vertex operator algebra associated to the lattice $K$. Since

$$
\left(e^{\alpha_{i 1}}+\cdots+e^{\alpha_{i l}}\right)_{-1}^{l-1}\left(e^{\alpha_{i 1}}+\cdots+e^{\alpha_{i l}}\right)=l ! e^{\alpha^{i 1}+\cdots+\alpha^{i l}}=l ! e^{\alpha^{i}}, i=1,2, \cdots, n-1,
$$

it follows that $e^{\alpha^{i}} \in L_{\widehat{\mathfrak{s l n}_{n}}}(l, 0), 1 \leq i \leq n-1$. Similarly, $e^{-\alpha^{i}} \in L_{\widehat{\mathfrak{s l}}_{n}}(l, 0), 1 \leq i \leq n-1$. Therefore $V_{K}$ is a vertex operator subalgebra of $L_{\widehat{\mathfrak{s l n}_{n}}}(l, 0)$. Hence every $L_{\widehat{\mathfrak{s n}_{n}}}(l, 0)$-module regarded as a $V_{K}$-module is completely reducible. By Lemma 2.8, every irreducible module of $V_{K}$ comes from $V_{K+\gamma}, \gamma \in K^{\circ} / K$. It is known that the lowest weight of $V_{K+\gamma}$ for $0 \neq \gamma \in K^{\circ} / K$ is positive. Note also that $\left\{v \in V_{K} \mid h_{m} v=0, h \in \mathfrak{h}_{n}, m \geq 0\right\}=\mathbb{C} 1$. Then we have

$$
K\left(\mathfrak{s l}_{n}, l\right)=\left\{v \in L_{\mathfrak{s l}_{n}}(l, 0) \mid u_{m} v=0, u \in V_{K}, m \geq 0\right\}
$$

and

$$
L_{\widehat{\mathfrak{s t}_{n}}}(l, 0)=\bigoplus_{\gamma \in K^{\circ} / K} V_{K+\gamma} \otimes U^{(l, n)}(0, \gamma),
$$

where $U^{(l, n)}(0,0)=K\left(\mathfrak{s l}_{n}, l\right)$ and $U^{(l, n)}(0, \gamma)$ for $\gamma \neq 0$ are modules of $K\left(\mathfrak{s l}_{n}, l\right)$ (also see [DW3]). More generally, let $L_{\widehat{\mathfrak{s l n}_{n}}}(l, \bar{\Lambda})$ be an irreducible module of the vertex operator algebra $L_{\widehat{\mathfrak{s l}}_{n}}(l, 0)$. Then we can decompose $L_{\widehat{\mathfrak{s}}_{n}}(l, \bar{\Lambda})$ as follows:

$$
L_{\widehat{\mathfrak{s} l_{n}}}(l, \bar{\Lambda})=\bigoplus_{\gamma \in K^{\circ} / K} V_{K+\bar{\Lambda}+\gamma} \otimes U^{(l, n)}(\bar{\Lambda}, \gamma)
$$

where $U^{(l, n)}(\bar{\Lambda}, \gamma)$ for $\gamma \in K^{\circ}$ are modules of $K\left(\mathfrak{s l}_{n}, l\right)$. We have the following lemma.

Lemma 3.4. (1) In (3.5) and (3.6), if $U^{(l, n)}(\bar{\Lambda}, \gamma) \neq 0$, then $U^{(l, n)}(\bar{\Lambda}, \gamma)$ is an irreducible $K\left(\mathfrak{s l}_{n}, l\right)$-module.

(2) $U^{(l, n)}(0,0)$ is not isomorphic to $U^{(l, n)}(\bar{\Lambda},-\bar{\Lambda})$ as $K\left(\mathfrak{s l}_{n}, l\right)$-modules, for any $\Lambda \neq 0$.

Proof. For $\gamma \in K^{\circ} / K$ such that $U^{(l, n)}(\bar{\Lambda}, \gamma) \neq 0$, let $W$ be a non-zero $K\left(\mathfrak{s l}_{n}, l\right)$-submodule of $U^{(l, n)}(\bar{\Lambda}, \gamma)$. Let $M$ be the $L_{\widehat{\mathfrak{s f n}}_{n}}(l, 0)$-submodule generated by $W$. For $\gamma_{1} \in K^{\circ} / K$, let $u \in V_{\bar{\Lambda}+\gamma_{1}} \otimes U^{(l, n)}\left(\bar{\Lambda}, \gamma_{1}\right), v \in V_{\bar{\Lambda}+\gamma} \otimes W$. Then by (2) of Lemma 2.8,

$$
u_{m} v \in V_{2 \bar{\Lambda}+\gamma_{1}+\gamma} \otimes U^{(l, n)}\left(2 \bar{\Lambda}+\gamma_{1}+\gamma\right), m \in \mathbb{Z} .
$$

Therefore $u_{m} v \in V_{\bar{\Lambda}+\gamma} \otimes U^{(l, n)}(\bar{\Lambda}+\gamma)$, for $m \in \mathbb{Z}$ if and only if $\bar{\Lambda}+\gamma_{1} \in K$. That is, $V_{\bar{\Lambda}+\gamma_{1}}=V_{K}$. Then we have $u_{m} v \in V_{\bar{\Lambda}+\gamma} \otimes W$. Hence

$$
M \cap\left(V_{\bar{\Lambda}+\gamma} \otimes U^{(l, n)}(\bar{\Lambda}+\gamma)\right)=V_{\bar{\Lambda}+\gamma} \otimes W .
$$

Since $L_{\widehat{\mathfrak{s l}_{n}}}(l, \bar{\Lambda})$ is simple, it follows that $W=U^{(l, n)}(\bar{\Lambda}, \gamma)$. (1) is proved.

Let $L_{\widehat{\mathfrak{s l}_{n}}}(l, \bar{\Lambda})$ be an irreducible module of the vertex operator algebra $L_{\widehat{\mathfrak{s l}}_{n}}(l, 0)$ such that $\Lambda \neq 0$. Then the lowest weight of $L_{\mathfrak{s f n}_{n}}(l, \bar{\Lambda})$ is positive ( see [LL] and also Lemma 12.8 in $[\mathrm{K}])$. Since $U^{(l, n)}(\bar{\Lambda},-\bar{\Lambda})$ as a $K\left(\mathfrak{s l}_{n}, l\right)$-module has the same lowest weight with $L_{\mathfrak{s f n}_{n}}(l, \bar{\Lambda})$, it follows that $U^{(l, n)}(0,0)$ can not be isomorphic to $U^{(l, n)}(\bar{\Lambda},-\bar{\Lambda})$, since the lowest weight of $U^{(l, n)}(0,0)$ as a $K\left(\mathfrak{s l}_{n}, l\right)$-module is zero. 
Note that as an $\mathfrak{s l}_{n}$-module, $V$ is integrable and the weight system of $V$ is a subset of the root lattice of $\mathfrak{s l}_{n}$. Then for each irreducible $\mathfrak{s l}_{n}$-submodule $W$ of $V$, there is a nonzero vector $u$ such that $h_{0} u=0$ for all $h \in \mathfrak{h}_{n}$. On the other hand, since $U=L_{\mathfrak{s l}_{n}}(l, 0)$ is rational, it follows that as an $L_{\widehat{\mathfrak{s}}_{n}}(l, 0)$-module, $V$ is completely reducible. So we have

$$
V=L_{\widehat{\mathfrak{s}_{n}}}(l, 0) \otimes C_{V}(U) \bigoplus\left(\bigoplus_{\lambda \in P_{+}^{n}, \lambda \neq l \Lambda_{0}} L_{\widehat{\mathfrak{s l}_{n}}}(l, \bar{\lambda}) \otimes M^{(l, n)}(\bar{\lambda})\right),
$$

where $P\left(l \Lambda_{0}\right)$ is the set of weights of $L_{\widehat{\mathfrak{s l n}_{n}}}(l, 0)$ and

$$
P_{+}^{n}=\left\{\lambda \in P\left(l \Lambda_{0}\right) \mid \lambda+\delta \notin P\left(l \Lambda_{0}\right),\left(\lambda, \alpha_{i}\right) \in \mathbb{Z}_{\geq 0}, i=0,1, \cdots, n-1\right\} .
$$

$\alpha_{0}, \cdots, \alpha_{n-1}$ are the simple roots of the affine Lie algebra $\widehat{\mathfrak{s l}_{n}}, \lambda=l \Lambda_{0}+\bar{\lambda}-m \delta$ for some $m \geq 0$ as introduced in $\S 6.2$ in $[\mathrm{K}]$, and $\left(\Lambda_{0}, \alpha_{i}\right)=\delta_{i 0}, i=0,1, \cdots, n-1 . M^{(l, n)}(\bar{\lambda}) \subseteq V_{N_{n}^{l}}$ are modules of $C_{V}(U), \lambda \in P_{+}^{n}$. As above considering $V$ as a module of the Heisenberg vertex operator algebra $L_{\widehat{h_{n}}}(l, 0)$, together with $(3.7)$ we have

$$
V_{N_{n}^{l}}=K\left(\mathfrak{s l}_{n}, l\right) \otimes C_{V}(U) \bigoplus\left(\bigoplus_{\lambda \in P_{+}^{n}, \lambda \neq l \Lambda_{0}} W^{(l, n)}(\bar{\lambda}) \otimes M^{(l, n)}(\bar{\lambda})\right),
$$

where we denote $W^{(l, n)}(\bar{\lambda})=U^{(l, n)}(\bar{\lambda},-\bar{\lambda})$. Following the similar argument for (3.4), we have

$$
U^{(l, n)}(\bar{\lambda},-\bar{\lambda})=\left\{v \in L_{\widehat{\mathfrak{s h}_{n}}}(l, \bar{\lambda}) \mid h_{m} v=0, h \in \mathfrak{h}_{n}\right\}
$$

Then by Lemma $3.4, W^{(l, n)}(\bar{\lambda})$ is an irreducible $K\left(\mathfrak{s l}_{n}, l\right)$-module. We have the following lemma.

Lemma 3.5. For $\lambda \in P_{+}^{n}, \lambda \neq l \Lambda_{0}$, let $M^{(l, n)}(\bar{\lambda})$ be as in (3.7). If $M^{(l, n)}(\bar{\lambda}) \neq 0$, then as a $C_{V}(U)$-module, the lowest weight of $M^{(l, n)}(\bar{\lambda})$ is a positive number.

Proof. For $\lambda \in P_{+}^{n}$, by Proposition 11.4 in $[\mathrm{K}]$, we have

$$
\left(l \Lambda_{0}+\rho, l \Lambda_{0}+\rho\right)-(\lambda+\rho, \lambda+\rho) \geq 0
$$

and the equality holds if and only if $\lambda=l \Lambda_{0}$, where $\left(\Lambda_{0}, \Lambda_{0}\right)=0,\left(\rho, \alpha_{i}\right)=1, i=$ $0,1, \cdots, n-1,\left(\rho, \Lambda_{0}\right)=0($ see $\oint 6.2$ in $[\mathrm{K}])$. Then we have for $\lambda \in P_{+}^{n}$ such that $\lambda<l \Lambda_{0}$,

$$
(\lambda+2 \rho, \lambda)<0 \text {. }
$$

Let $\omega^{\prime}$ be the conformal vector of $L_{\mathfrak{s l n}_{n}}(l, 0)$, and $\omega^{\prime \prime}=\omega-\omega^{\prime}$ the conformal vector of $C_{V}(U)$. For $\lambda \in P_{+}^{n}, \lambda \neq l \Lambda_{0}$, denote

$$
M^{(l, n)}(\bar{\lambda})=\bigoplus_{\substack{m \in \mathbb{Q} \\ m \geq a(\bar{\lambda})}} M^{(l, n)}(\bar{\lambda})_{(m)}
$$

such that

$$
\omega_{1}^{\prime \prime}\left(M^{(l, n)}(\bar{\lambda})_{(m)}\right)=\left.\operatorname{mid}\right|_{M^{(l, n)}(\bar{\lambda})_{(m)}}
$$


where $a(\bar{\lambda})$ is a rational number. Then by Corollary 12.8 in $[\mathrm{K}]$ we have

$$
a(\bar{\lambda})=-\frac{(\lambda+2 \rho, \lambda)}{2(l+n)}>0 .
$$

Let

$$
\widetilde{A}_{l-1}^{\times n}=\bigoplus_{i=1}^{l-1} \bigoplus_{j=1}^{n} \mathbb{Z} \beta^{i j}
$$

be an even lattice such that for $1 \leq i, p \leq l-1,1 \leq j, q \leq n$,

$$
\begin{gathered}
\left(\beta^{i j}, \beta^{p q}\right)=0, \text { if } j \neq q, \\
\left(\beta^{i j}, \beta^{p j}\right)= \begin{cases}2, & \text { if } p=i, \\
-1, & \text { if } i=p-1 \text { or } i=p+1, \\
0 & \text { otherwise. }\end{cases}
\end{gathered}
$$

Set

$$
\widetilde{N}_{l}^{n}=\bigoplus_{i=1}^{l-1} \bigoplus_{j=1}^{n-1} \mathbb{Z}\left(\beta^{i j}-\beta^{i, j+1}\right)
$$

Then

$$
V_{\widetilde{N}_{l}^{n}}=\left\{v \in V_{\widetilde{A}_{l-1}^{\times n}} \mid \sum_{j=1}^{n} \beta^{i j}(m) v=0,1 \leq i \leq l-1, m \geq 0\right\} .
$$

For $1 \leq i \leq l-1$, let

$$
\begin{gathered}
\widetilde{\omega}^{i}=\frac{1}{n+2} \sum_{1 \leq p<q \leq n}\left[\frac{1}{2 n}\left(\beta^{i p}-\beta^{i q}\right)(-1)^{2} \mathbf{1}+\left(e^{\beta^{i p}-\beta^{i q}}+e^{-\beta^{i p}+\beta^{i q}}\right)\right], \\
\widetilde{W}^{3, i}=\sum_{1 \leq p, q, r \leq n}\left(\beta^{i p}-\beta^{i q}\right)(-1)^{2}\left(\beta^{i p}-\beta^{i r}\right)(-1) \mathbf{1} \\
\left.-3 n \sum_{1 \leq q, r \leq n, q \neq r}\left[\sum_{1 \leq p \leq n, p \neq q}\left(\beta^{i p}-\beta^{i q}\right)(-1)+\sum_{1 \leq p \leq n, p \neq r}\left(\beta^{i p}-\beta^{i r}\right)\right)(-1)\right] e^{\beta^{i q}-\beta^{i r}} .
\end{gathered}
$$

The following lemma follows from [DLY2], [DLWY] and [DW1].

Lemma 3.6. (1) For every $i$ such that $1 \leq i \leq l-1, \widetilde{W}^{3, i}$ generates a simple vertex operator algebra isomorphic to the parafermion vertex operator algebra $K\left(\mathfrak{s l}_{2}, n\right)$ with $\widetilde{\omega}^{i}$ as its Virasoro vector.

(2) The vertex operator algebra generated by $\widetilde{W}^{3, i}, 1 \leq i \leq l-1$ is isomorphic to the parafermion vertex operator algebra $K\left(\mathfrak{s l}_{l}, n\right)$.

For $1 \leq i \leq l-1$, set

$$
\begin{aligned}
\omega^{i}= & \frac{1}{n+2} \sum_{1 \leq p \leq q \leq n-1}\left[\frac{1}{2 n}\left(\alpha^{p i}-\alpha^{p, i+1}+\alpha^{p+1, i}-\alpha^{p+1, i+1}+\cdots+\alpha^{q i}-\alpha^{q, i+1}\right)(-1)^{2} \mathbf{1}\right. \\
& \left.+(-1)^{p-q+1}\left(e^{\alpha^{p i}-\alpha^{p, i+1}+\cdots+\alpha^{q i}-\alpha^{q, i+1}}+e^{-\left(\alpha^{p i}-\alpha^{p, i+1}+\cdots+\alpha^{q i}-\alpha^{q, i+1}\right)}\right)\right],
\end{aligned}
$$




$$
\begin{aligned}
W^{3, i}=- & \sum_{p=1}^{n}\left[\sum_{q=1}^{p-1}\left(\alpha^{q i}-\alpha^{q, i+1}+\alpha^{q+1, i}-\alpha^{q+1, i+1}+\cdots+\alpha^{p-1, i}-\alpha^{p-1, i+1}\right)(-1)^{2}\right. \\
& \left.+\sum_{q=p}^{n-1}\left(\alpha^{p i}-\alpha^{p, i+1}+\cdots+\alpha^{q i}-\alpha^{q i+1}\right)(-1)^{2}\right]\left[-\sum_{k=1}^{p-1} k\left(\alpha^{k i}-\alpha^{k, i=1}\right)(-1) \mathbf{1}\right. \\
& +\sum_{k=p}^{n-1}(n-k)\left(\alpha^{k i}-\alpha^{k j}(-1) \mathbf{1}\right]+3 n \sum_{q=1}^{n} \sum_{r=1}^{q-1}(-1)^{q-r}\left[\sum_{k=1}^{q-1} k\left(\alpha^{k i}-\alpha^{k, i+1}\right)(-1)\right. \\
& -\sum_{k=q}^{n-1}(n-k)\left(\alpha^{k i}-\alpha^{k, i+1}\right)(-1)+\sum_{k=1}^{r-1} k\left(\alpha^{k i}-\alpha^{k, i+1}\right)(-1) \\
& \left.-\sum_{k=r}^{n-1}(n-k)\left(\alpha^{k i}-\alpha^{k, i+1}\right)(-1)\right] e^{-\sum_{k=r}^{q-1}\left(\alpha^{k i}-\alpha^{k, i+1}\right)} \\
& +3 n \sum_{q=1}^{n} \sum_{r=q+1}^{n}(-1)^{q-r}\left[\sum_{k=1}^{q-1} k\left(\alpha^{k i}-\alpha^{k, i+1}\right)(-1)-\sum_{k=q}^{n-1}(n-k)\left(\alpha^{k i}-\alpha^{k, i+1}\right)(-1)\right. \\
& \left.+\sum_{k=1}^{r-1} k\left(\alpha^{k i}-\alpha^{k, i+1}\right)(-1)-\sum_{k=r}^{n-1}(n-k)\left(\alpha^{k i}-\alpha^{k, i+1}\right)(-1)\right] e^{\sum_{k=q}^{r-1}\left(\alpha^{k i}-\alpha^{k, i+1}\right)} .
\end{aligned}
$$

It is known that for $l=2, C_{V}(U)$ is isomorphic to the parafermion vertex operator algebra $K\left(\mathfrak{s l}_{2}, n\right)$ (see [CGT] and the introduction in [DLY1]). The following lemma gives a concrete description of the conformal vector and the weight 3 generator of $C_{V}(U)$ for $l=2$.

Lemma 3.7. Let $l=2$. Then the vertex operator algebra $C_{V}(U)$ is generated by $W^{3,1}$, and $\omega^{1}$ is the conformal vector of $C_{V}(U)$.

Proof. Consider the lattice vertex operator algebras $V_{\widetilde{A}_{1}^{\times n}}$ and $V_{\widetilde{N}_{2}^{n}}$. Note that $V_{\widetilde{A}_{1}^{\times n}}$ is

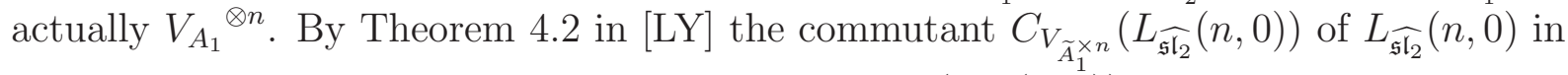
$V_{\widetilde{A}_{1}^{\times n}}$ is contained in $V_{\widetilde{N}_{2}^{n}}$ and the commutant of $C_{V_{\widetilde{A}_{1}^{\times n}}}\left(L_{\widehat{\mathfrak{s l}}_{2}}(n, 0)\right)$ in $V_{\widetilde{N}_{2}^{n}}$ is the parafermion vertex operator algebra $K\left(\mathfrak{s l}_{2}, n\right)$ generated by $\widetilde{\omega}^{1}$ and $\widetilde{W}^{3,1}$. Furthermore,

$$
V_{\widetilde{N}_{2}^{n}}=C_{V_{\widetilde{A}_{1}^{\times n}}}\left(L_{\widehat{\mathfrak{s l}_{2}}}(n, 0)\right) \otimes K\left(\mathfrak{s l}_{2}, n\right) \bigoplus\left(\bigoplus_{\substack{2 \leq k_{n-1} \leq n \\ k_{n-1} \in 2 \mathbb{Z}}} M\left(k_{n-1}\right) \otimes W\left(k_{n-1}\right)\right),
$$

where $M\left(k_{n-1}\right), 2 \leq k_{n-1} \leq n$ are irreducible modules of $C_{V_{\widetilde{A}_{1}^{\times n}}}\left(L_{\widehat{\mathfrak{s l}_{2}}}(n, 0)\right)$ and $W\left(k_{n-1}\right), 2 \leq$

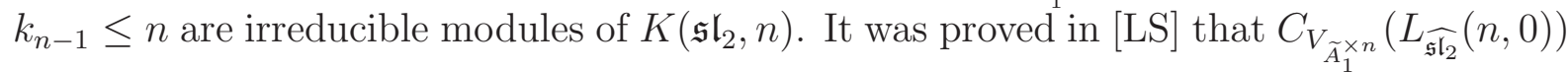
is generated by

$$
u^{i j}=\frac{1}{16}\left(\beta^{1 i}-\beta^{1, j+1}\right)(-1)^{2} \mathbf{1}-\frac{1}{4}\left(e^{\beta^{1 i}-\beta^{1, j+1}}+e^{-\beta^{1 i}+\beta^{1, j+1}}\right), 1 \leq i \leq j \leq n-1 .
$$

For $1 \leq i \leq j \leq n-1$, set

$$
v^{i j}=\frac{1}{16}\left(\sum_{k=i}^{j}\left(\alpha^{k 1}-\alpha^{k 2}\right)\right)(-1)^{2} \mathbf{1}-\frac{1}{4}\left(e^{\sum_{k=i}^{j}\left(\alpha^{k 1}-\alpha^{k 2}\right)}+e^{\sum_{k=i}^{j}\left(-\alpha^{k 1}+\alpha^{k 2}\right)}\right) .
$$


Note that both $N_{n}^{2}=\bigoplus_{i=1}^{n-1} \mathbb{Z}\left(\alpha^{i 1}-\alpha^{i 2}\right)$ and $\widetilde{N}_{2}^{n}=\bigoplus_{i=1}^{n-1} \mathbb{Z}\left(\beta^{1 i}-\beta^{1, i+1}\right)$ are lattice of type $\sqrt{2} A_{n-1}$ with bases $\left\{\alpha^{i 1}-\alpha^{i 2} \mid i=1,2, \cdots, n-1\right\}$ and $\left\{\beta^{1 i}-\beta^{1, i+1} \mid 1=\right.$ $1,2, \cdots, n-1\}$ respectively. It follows that $V_{N_{n}^{2}}$ and $V_{\widetilde{N}_{2}^{n}}$ are isomorphic and the vertex operator algebra generated by $v^{i j}, 1 \leq i \leq j \leq n-1$ is isomorphic to the vertex operator algebra generated by $u^{i j}, 1 \leq i \leq j \leq n-1$ with the isomorphism $\sigma$ : $v^{i j} \mapsto u^{i j}$, $1 \leq i \leq j \leq n-1$. Let $\theta$ be an automorphism of the vertex operator algebra $V_{N_{2}^{n}}$ defined by

$$
\begin{gathered}
\theta\left(e^{\alpha^{k 1}-\alpha^{k 2}}\right)=-e^{-\alpha^{k 1}+\alpha^{k 2}}, \theta\left(e^{-\alpha^{k 1}+\alpha^{k 2}}\right)=-e^{\alpha^{k 1}-\alpha^{k 2}}, \\
\theta\left(\left(\alpha^{k 1}-\alpha^{k 2}\right)(-1) \mathbf{1}\right)=\left(-\alpha^{k 1}+\alpha^{k 2}\right)(-1) \mathbf{1}, 1 \leq k \leq n-1 .
\end{gathered}
$$

Then

$$
\begin{gathered}
\theta\left(\sigma^{-1}\left(u^{i i}\right)\right)=\theta\left(v^{i i}\right)={ }^{\prime} \omega^{i}, i=1,2, \cdots, n-1, \\
\theta\left(\sigma^{-1}\left(\widetilde{\omega}^{1}\right)\right)=\omega^{1}, \theta\left(\sigma^{-1}\left(\widetilde{W^{3,1}}\right)\right)=W^{3,1},
\end{gathered}
$$

where ${ }^{\prime} \omega^{i}$ is defined by (3.3) for $l=2$. Then we see that $C_{V}(U)=\theta\left(\sigma^{-1}(K(\mathfrak{s l} 2, n))\right)$ and $C_{V}(U)$ is generated by $\omega^{1}$ and $W^{3,1}$.

Since $U \cong L_{\widehat{\mathfrak{s l}_{n}}}(2,0)$ for $l=2$, it follows from $(3.3)$ that ${ }^{\prime} \omega^{i}, 1 \leq i \leq n-1$ are in $K\left(\mathfrak{s l}_{n}, 2\right)$. By Lemma 3.6 the vertex operator subalgebra of $U$ generated by ' $\omega^{i}, 1 \leq i \leq n-1$ is isomorphic to the parafermion vertex operator algebra $K\left(\mathfrak{s l}_{n}, 2\right)$. By Proposition 4.6 in [LS] ( also see [JL]), $C_{V_{\widetilde{A}_{1}^{\times n}}}\left(L_{\widehat{\mathfrak{s l}}_{2}}(n, 0)\right)$ is generated by $u^{i i}, i=1,2, \cdots, n-1$. Then using the maps $\theta^{-1}$ and $\sigma$, we obtain the following lemma.

Lemma 3.8. $C_{V_{\widetilde{A}_{1}^{\times n}}}\left(L_{\widehat{\mathfrak{s l}}}(n, 0)\right)$ is isomorphic to the parafermion vertex operator algebra $K\left(\mathfrak{s l}_{n}, 2\right)$, and

$$
V_{\widetilde{N}_{2}^{n}}=K\left(\mathfrak{s l}_{n}, 2\right) \otimes K\left(\mathfrak{s l}_{2}, n\right) \bigoplus\left(\bigoplus_{\substack{2 \leq k_{n-1} \leq n \\ k_{n-1} \in 2 \mathbb{Z}}} M\left(k_{n-1}\right) \otimes W\left(k_{n-1}\right)\right) .
$$

For $n, l \in \mathbb{Z}_{\geq 3}$, let $W(0)$ be the vertex operator subalgebra of $V_{N_{n}^{l}}$ generated by $\omega^{i}$ and $W^{3, i}, 1 \leq i \leq l-1$ and $\widetilde{W}(0)$ the vertex operator subalgebra of $V_{\widetilde{N}_{l}^{n}}$ generated by $\widetilde{\omega}^{i}$ and $\widetilde{W}^{3, i}, 1 \leq i \leq l-1$. Then we have the following lemma.

Lemma 3.9. (1) There is a vertex operator algebra isomorphism $\tau$ from $V_{N_{n}^{l}}$ to $V_{\widetilde{N}_{l}^{n}}$ such that $\tau(W(0))=\widetilde{W}(0)$.

(2) Both $W(0)$ and $\widetilde{W}(0)$ are isomorphic to the parafermion vertex operator algebra $K\left(\mathfrak{s l}_{l}, n\right)$.

Proof. It is easy to check that for $1 \leq i, j \leq n-1,1 \leq p, q \leq l-1$,

$$
\left(\alpha^{i p}-\alpha^{i, p+1}, \alpha^{j q}-\alpha^{j, q+1}\right)=\left(\beta^{p i}-\beta^{p, i+1}, \beta^{q j}-\beta^{q, j+1}\right) .
$$


Then there is a vertex operator algebra isomorphism $\tau^{1}$ from $V_{N_{n}^{l}}$ to $V_{\widetilde{N}_{l}^{n}}$ such that

$$
\tau^{1}\left(\left(\alpha^{i p}-\alpha^{i, p+1}\right)(-1) \mathbf{1}\right)=\left(\beta^{p i}-\beta^{p, i+1}\right)(-1) \mathbf{1}, 1 \leq i \leq n-1,1 \leq p \leq l-1
$$

and

$$
\tau^{1}\left(e^{\alpha^{i p}-\alpha^{i, p+1}}\right)=e^{\beta^{p i}-\beta^{p, i+1}}, 1 \leq i \leq n-1,1 \leq p \leq l-1 .
$$

Let $\theta$ be an automorphism of $V_{N_{n}^{l}}$ defined by

$$
\begin{gathered}
\theta\left(e^{\alpha^{i p}-\alpha^{i, p+1}}\right)=-e^{-\alpha^{i p}+\alpha^{i, p+1}}, \theta\left(e^{-\alpha^{i p}+\alpha^{i, p+1}}\right)=-e^{\alpha^{i p}-\alpha^{i, p+1}}, \\
\theta\left(\left(\alpha^{i p}-\alpha^{i, p+1}\right)(-1) \mathbf{1}\right)=\left(-\alpha^{i p}+\alpha^{i, p+1}\right)(-1) \mathbf{1}, 1 \leq i \leq n-1,1 \leq p \leq l-1 .
\end{gathered}
$$

Then $\tau=\tau^{1} \theta$ is the desired vertex operator algebra isomorphism from $V_{N_{n}^{l}}$ to $V_{\widetilde{N}_{l}^{n}}$. By Lemma 3.6, $\widetilde{W}(0)$ is isomorphic to the parafermion vertex operator algebra $K\left(\mathfrak{s l}_{l}, n\right)$. Since $\widetilde{W}(0)=\tau(W(0))$, it follows that $W(0)$ is also isomorphic to $K\left(\mathfrak{s l}_{l}, n\right)$.

We are now in a position to state the following level-rank duality which characterizes relations between tensor decomposition and parafermion vertex operator algebras.

Theorem 3.10. For $n, l \in \mathbb{Z}_{\geq 2}, V=L_{\widehat{\mathfrak{s l}}_{n}}(1,0)^{\otimes l}$ and $U=L_{\widehat{\mathfrak{s l n}_{n}}}(l, 0)$, we have

$$
\begin{gathered}
V=L_{\mathfrak{s l}_{n}}(l, 0) \otimes K\left(\mathfrak{s l}_{l}, n\right) \bigoplus\left(\bigoplus_{\lambda \in P_{+}^{n}, \lambda \neq l \Lambda_{0}} L_{\widehat{\mathfrak{s}}_{n}}(l, \bar{\lambda}) \otimes M^{(l, n)}(\bar{\lambda})\right), \\
V_{N_{n}^{l}}=K\left(\mathfrak{s l}_{n}, l\right) \otimes K\left(\mathfrak{s l}_{l}, n\right) \bigoplus\left(\bigoplus_{\lambda \in P_{+}^{n}, \lambda \neq l \Lambda_{0}} W^{(n, l)}(\bar{\lambda}) \otimes M^{(l, n)}(\bar{\lambda})\right),
\end{gathered}
$$

where if $M^{(l, n)}(\bar{\lambda}) \neq 0$, then $M^{(l, n)}(\bar{\lambda})$ as a $K\left(\mathfrak{s l}_{l}, n\right)$-module is isomorphic to $W^{(n, l)}(\bar{\mu})$ for some $\mu \in P_{+}^{l} . P\left(n \Lambda_{0}\right)$ is the set of weights of $L_{\widehat{s l}_{l}}(n, 0)$ and

$$
P_{+}^{l}=\left\{\lambda \in P\left(n \Lambda_{0}\right) \mid \lambda+\delta \notin P\left(n \Lambda_{0}\right),\left(\lambda, \beta_{i}\right) \in \mathbb{Z}_{\geq 0}, i=0,1, \cdots, l-1\right\},
$$

where $\beta_{0}, \cdots, \beta_{l-1}$ are the simple roots of the affine Lie algebra $\widehat{\mathfrak{s l}}_{l}$.

Proof. By Lemma 3.8, we may assume that $n, l \geq 3$. Let $W(0)$ and $\widetilde{W}(0)$ be defined as above. By Lemma 3.7, it is easy to see that $\omega^{i}, W^{3, i} \in C_{V}(U), 1 \leq i \leq l-1$. Then by Lemma 3.9, $K\left(\mathfrak{s l}_{l}, n\right) \cong W(0) \subseteq C_{V_{A_{n-1}^{\times l}}}\left(L_{\widehat{\mathfrak{s l}_{n}}}(l, 0)\right)$. By (3.7) and (3.8), we have

$$
\begin{gathered}
V=L_{\widehat{s i}_{n}}(l, 0) \otimes C_{V}(U) \bigoplus\left(\bigoplus_{\lambda \in P_{+}^{n}, \lambda \neq l \Lambda_{0}} L_{\widehat{\mathfrak{s l}}_{n}}(l, \bar{\lambda}) \otimes M^{(l, n)}(\bar{\lambda})\right), \\
V_{N_{n}^{l}}=K\left(\mathfrak{s l}_{n}, l\right) \otimes C_{V}(U) \bigoplus\left(\bigoplus_{\lambda \in P_{+}^{n}, \lambda \neq l \Lambda_{0}} W^{(l, n)}(\lambda) \otimes M^{(l, n)}(\bar{\lambda})\right) .
\end{gathered}
$$

Regarding $V_{N_{n}^{l}}$ as a $C_{V}(U)$-module, by Lemma 3.5,

$$
V_{N_{n}^{l}}(0)=\left\{v \in V_{N_{n}^{l}} \mid \omega_{1}^{\prime \prime} v=0\right\}=K\left(\mathfrak{s l}_{n}, l\right),
$$


where $\omega^{\prime \prime}$ is the conformal vector of $C_{V}(U)$. Since $K\left(\mathfrak{s l}_{l}, n\right) \cong W(0)$ is a conformal vertex operator subalgebra of $C_{V}(U)$, it follows that regarding $V_{N_{n}^{l}}$ as a $W(0)$-module, we also have $V_{N_{n}^{l}}(0)=K\left(\mathfrak{s l}_{n}, l\right)$.

Consider $V_{\widetilde{A}_{l-1}^{\times n}}$ and $V_{\widetilde{N}_{l}^{n}}$ similarly, we have $K\left(\mathfrak{s l}_{n}, l\right) \subseteq C_{V_{\widetilde{A}_{l-1}^{\times n}}}\left(L_{\mathfrak{s l}_{l}}(n, 0)\right)$ and

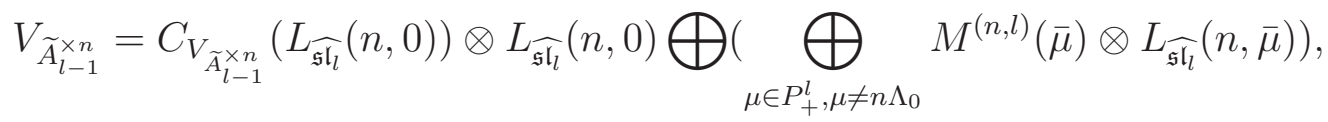

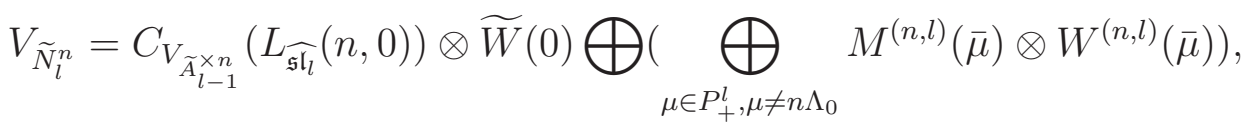

where $P\left(n \Lambda_{0}\right)$ is the set of weights of $L_{\widehat{\mathfrak{s l}_{l}}}(n, 0)$, and

$$
P_{+}^{l}=\left\{\lambda \in P\left(n \Lambda_{0}\right) \mid \lambda+\delta \notin P\left(n \Lambda_{0}\right),\left(\lambda, \beta_{i}\right) \in \mathbb{Z}_{\geq 0}, i=0,1, \cdots, l-1\right\},
$$

$\beta_{0}, \cdots, \beta_{l-1}$ are the simple roots of the affine Lie algebra $\widehat{\mathfrak{s l}}_{l}$.

Recall that $\widetilde{W}(0) \cong K\left(\mathfrak{s l}_{l}, n\right)$. Regarding $V_{\widetilde{N}_{l}^{n}}$ as a $\widetilde{W}(0)$-module, by $(3.14)$ we have

$$
V_{\tilde{N}_{l}^{n}}(0)=\left\{v \in V_{\widetilde{N}_{l}^{n}} \mid \widetilde{\omega}_{1}^{\prime \prime} v=0\right\} \supseteq C_{V_{\widetilde{A}_{l-1}^{\times n}}}\left(L_{\widehat{\mathfrak{s l}}_{l}}(n, 0)\right),
$$

where $\widetilde{\omega}^{\prime \prime}$ is the Virasoro vector of $\widetilde{W}(0)$ inside $V_{\widetilde{N}_{l}^{n}}$. By Lemma 3.9, $V_{\widetilde{N}_{l}^{n}} \cong V_{N_{n}^{l}}$ through the isomorphism $\tau$ and $\tau(W(0))=\widetilde{W}(0)$. By (3.13), we immediately have

$$
C_{V_{\widetilde{A}_{l-1}^{\times n}}}\left(L_{\widehat{\mathfrak{s l} l}}(n, 0)\right)=K\left(\mathfrak{s l}_{n}, l\right) .
$$

Similarly,

$$
C_{V_{A_{n-1}^{\times l}}}\left(L_{\widehat{\mathfrak{s l}_{n}}}(l, 0)\right)=C_{V}(U)=K\left(\mathfrak{s l}_{l}, n\right) .
$$

\section{Level-Rank Duality for General Case}

In this section, we will establish the level-rank duality for general case. As in Section 3 , let $V$ be the tensor product vertex operator algebra

$$
V=L_{\widehat{\mathfrak{g}}}\left(l_{1}, 0\right) \otimes L_{\widehat{\mathfrak{g}}}\left(l_{2}, 0\right) \otimes \cdots \otimes L_{\widehat{\mathfrak{g}}}\left(l_{m}, 0\right)
$$

and $U \cong L_{\widehat{\mathfrak{s l}}_{n}}(l, 0)$ the vertex operator subalgebra of $V$ generated by $\mathfrak{s l}_{n}(\mathbb{C})$ which is diagonally imbedded into $V_{1}$, where $\mathfrak{g}=\mathfrak{s l}_{n}(\mathbb{C}), l_{1}, \cdots, l_{m} \in \mathbb{Z}_{+}, l=l_{1}+\cdots+l_{m}$, $m \in \mathbb{Z}_{\geq 2}$.

Let $A_{n-1}^{\times l}, \widetilde{A}_{l-1}^{\times n}, N_{n}^{l}$, and $\widetilde{N}_{l}^{n}$ be the same as in Section 3. Denote

$$
s_{0}=0, s_{j}=l_{1}+l_{2}+\cdots+l_{j}, 1 \leq j \leq m .
$$


For $r=1,2, \cdots, m$, define

$$
A_{n}^{\times l, r}=\bigoplus_{i=1}^{n-1} \bigoplus_{j=s_{r-1}+1}^{s_{r}} \mathbb{Z} \alpha^{i j},
$$

For $r=1,2, \cdots, m$ such that $s_{r} \geq 2$, define

$$
\begin{gathered}
\widetilde{A}_{l, r}^{\times n}=\bigoplus_{i=s_{r-1}+1}^{s_{r}-1} \bigoplus_{j=1}^{n} \mathbb{Z} \beta^{i j}, \\
N_{n}^{l, r}=\bigoplus_{i=1}^{n-1} \bigoplus_{j=s_{r-1}+1}^{s_{r}-1} \mathbb{Z}\left(\alpha^{i j}-\alpha^{i, j+1}\right), \quad \widetilde{N}_{l, r}^{n}=\bigoplus_{i=s_{r-1}+1}^{s_{r}-1} \bigoplus_{j=1}^{n-1} \mathbb{Z}\left(\beta^{i j}-\beta^{i, j+1}\right), \\
K_{r}=\bigoplus_{i=s_{r-1}+1}^{s_{r}-1} \mathbb{Z}\left(\beta^{i 1}+\cdots+\beta^{i n}\right) .
\end{gathered}
$$

Recall that the simple vertex operator algebra $L_{\widehat{\mathfrak{s l n}}_{n}}(l, 0)$ is naturally imbedded into the lattice vertex operator algebra $V_{A_{n-1}^{\times l}}$ and $L_{\widehat{\mathfrak{s l} l}}(n, 0)$ is naturally imbedded into $V_{A_{l-1}^{\times n}}$. We now imbed $L_{\widehat{\mathfrak{s l}_{n}}}\left(l_{k}, 0\right)$ into the vertex operator algebra $V_{A_{n}^{\times l, k}}$. For $l_{k} \geq 2$, we also imbed $L_{\widehat{\mathfrak{s l} l_{k}}}(n, 0)$ into $V_{\widetilde{A}_{l, k}^{\times n}}$.

Denote

$$
\mathfrak{h}_{l}=\bigoplus_{i=1}^{l-1} \mathbb{C}\left(\beta^{i 1}+\cdots+\beta^{i n}\right) .
$$

Then $\mathfrak{h}_{l}$ is a Cartan subalgebra of $L_{\widehat{\mathfrak{s l}_{l}}}(n, 0)$. Set

$$
\mathfrak{h}_{\underline{\ell}}=\left\{\beta \in \mathfrak{h}_{l} \mid\left(\beta, \beta^{i 1}+\cdots+\beta^{i n}\right)=0 \text {, for } s_{k} \geq 2, s_{k-1}+1 \leq i \leq s_{k}-1,1 \leq k \leq m\right\}
$$

and

$$
\mathfrak{l}_{\underline{\underline{\ell}}}=\left(\bigoplus_{k=1, l_{k} \geq 2}^{m} L_{\widetilde{\mathfrak{s l}}_{\mathfrak{l}_{k}}}(n, 0)_{1}\right) \bigoplus \underline{\mathfrak{h}_{\underline{\ell}}} .
$$

Then $\mathfrak{l}_{\underline{\ell}}$ is a Levi subalgebra of $\mathfrak{s l}_{l}(\mathbb{C})$ and $\mathfrak{h}_{\underline{\underline{\ell}}}$ is the center of $\mathfrak{l}_{\underline{\ell}}$ which is contained in the (fixed) Cartan subalgebra of $\mathfrak{s l}_{l}$. Denote by $L_{\widehat{\underline{\underline{\underline{r}}}}}(n, 0)$ the vertex operator subalgebra of $L_{\widehat{\mathfrak{s l}} \underline{l}}(n, 0)$ generated by $\mathfrak{l}_{\underline{\underline{\ell}}}$. It is easy to see that

$$
L_{\widehat{\widehat{\underline{\underline{e}}}}}(n, 0) \cong\left(\bigotimes_{k=1, l_{k} \geq 2}^{m} L_{\widehat{\mathfrak{s l l}_{l_{k}}}}(n, 0)\right) \bigotimes L_{\underline{\widehat{\mathfrak{h}}}_{\underline{\underline{e}}}}(n, 0),
$$

where $L_{\widehat{\mathfrak{h}} \underline{\underline{\ell}}}(n, 0)$ is the Heisenberg vertex operator subalgebra of $L_{\widehat{\mathfrak{s} l_{l}}}(n, 0)$ generated by $\mathfrak{h}_{\underline{\ell}}$. We denote

$$
K\left(\mathfrak{s l}_{l}, \underline{\mathfrak{l}_{\underline{\ell}}}, n\right)=C_{L_{\widehat{s} l_{l}}(n, 0)}\left(L_{\widehat{\widehat{l}}_{\underline{\underline{\ell}}}}(n, 0)\right) .
$$

Definition 4.1. $K\left(\mathfrak{s l}_{l}, \mathfrak{l}_{\underline{\underline{l}}}, n\right)$ is called a relative parafermion vertex operator algebra. 
We have the following level-rank duality which characterizes relations between tensor decomposition and relative parafermion vertex operator algebras.

Theorem 4.2. We have

$$
C_{L_{\widehat{\mathfrak{s l n}}}\left(l_{1}, 0\right) \otimes \cdots \otimes L_{\widehat{\mathfrak{s l n}}}\left(l_{m}, 0\right)}\left(L_{\widehat{\mathfrak{s l} n}}(l, 0)\right) \cong K\left(\mathfrak{s l}_{l}, \mathfrak{l}_{\underline{\ell}}, n\right)
$$

Proof. We just prove the theorem for $m=2$, since for $m>2$ the argument is quite similar. Now assume that $m=2$. Then $l=l_{1}+l_{2}$. We may assume that $l_{1}, l_{2} \geq 2$. Recall the natural imbedding

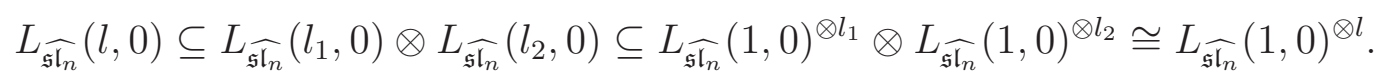

Let $P\left(l_{i} \Lambda_{0}\right)$ be the set of weights of the $\widehat{\mathfrak{s l}}_{n}$-module $L_{\widehat{\mathfrak{s l}_{n}}}\left(l_{i}, 0\right), i=1,2$. Denote

$$
P_{+}^{n, l_{i}}=\left\{\lambda \in P\left(l_{i} \Lambda_{0}\right) \mid \lambda \text { is integral dominate and } \lambda+\delta \notin P\left(l_{i} \Lambda_{0}\right)\right\}, i=1,2 .
$$

By Theorem 3.10, we have

$$
\begin{aligned}
& L_{\widehat{\mathfrak{s l}_{n}}}(1,0)^{\otimes l_{1}} \otimes L_{\widehat{\mathfrak{s} l_{n}}}(1,0)^{\otimes l_{2}} \\
& \quad=\left[\bigoplus_{\lambda \in P_{+}^{n, l_{1}}}\left(L_{\widehat{\mathfrak{s} l_{n}}}\left(l_{1}, \bar{\lambda}\right) \otimes M^{\left(l_{1}, n\right)}(\bar{\lambda})\right)\right] \otimes\left[\bigoplus_{\mu \in P_{+}^{n, l_{2}}}\left(L_{\widehat{\mathfrak{s l}_{n}}}\left(l_{2}, \bar{\mu}\right) \otimes M^{\left(l_{2}, n\right)}(\bar{\mu})\right)\right] \\
& \quad=\bigoplus_{\lambda \in P_{+}^{n, l_{1}}} \bigoplus_{\mu \in P_{+}^{n, l_{2}}}\left[L_{\widehat{\mathfrak{s l} n}}\left(l_{1}, \bar{\lambda}\right) \otimes L_{\widehat{\mathfrak{s l}_{n}}}\left(l_{2}, \bar{\mu}\right) \otimes M^{\left(l_{1}, n\right)}(\bar{\lambda}) \otimes M^{\left(l_{2}, n\right)}(\bar{\mu})\right] \\
& \quad=\bigoplus_{\lambda \in P_{+}^{n, l_{1}}} \bigoplus_{\mu \in P_{+}^{n, l_{2}}}\left[\bigoplus_{\gamma \in P_{+}(\lambda, \mu)}\left(L_{\widehat{\mathfrak{s l}}_{n}}(l, \bar{\gamma}) \otimes M(\lambda, \mu, \bar{\gamma})\right)\right] \otimes M^{\left(l_{1}, n\right)}(\bar{\lambda}) \otimes M^{\left(l_{2}, n\right)}(\bar{\mu}),
\end{aligned}
$$

where $P_{+}(\lambda, \mu)$ is a set of integral dominant weights of the $\widehat{\mathfrak{s l}}_{n}$-module $L_{\widehat{\mathfrak{s l}}_{n}}\left(l_{1}, \lambda\right) \otimes$ $L_{\widehat{\mathfrak{s l}}_{n}}\left(l_{2}, \mu\right) . \quad M(0,0,0)$ is the commutant vertex operator algebra $C_{V}(U)$ and $M(\lambda, \mu, \bar{\gamma})$ are $C_{V}(U)$-modules, $\lambda \in P_{+}^{n, l_{1}}, \mu \in P_{+}^{n, l_{2}}, \gamma \in P_{+}(\lambda, \mu)$. So

$$
C_{L_{\widehat{\mathfrak{s} n} n}(1,0) \otimes l}\left(L_{\widehat{\mathfrak{s l n}}}(l, 0)\right)=\bigoplus_{\lambda \in P_{+}^{n, l_{1}}} \bigoplus_{\mu \in P_{+}^{n, l_{2}}} M^{\left(l_{1}, n\right)}(\bar{\lambda}) \otimes M^{\left(l_{2}, n\right)}(\bar{\mu}) \otimes M(\lambda, \mu, 0) .
$$

Note that

$$
M^{\left(l_{1}, n\right)}(0)=C_{L_{\widehat{\mathfrak{s l n}}}(1,0)^{\otimes l_{1}}}\left(L_{\widehat{\mathfrak{s l}_{n}}}\left(l_{1}, 0\right)\right), M^{\left(l_{2}, n\right)}(0)=C_{L_{\widehat{\mathfrak{s} l_{n}}}(1,0)^{\otimes l_{2}}}\left(L_{\widehat{\mathfrak{s l}_{n}}}\left(l_{2}, 0\right)\right) .
$$

On the other hand, we have

$$
\begin{aligned}
L_{\widehat{\mathfrak{s l} l}}(n, 0)= & \bigoplus_{\lambda^{1}, \lambda^{2}, \lambda^{3}} L_{\widehat{\mathfrak{s l} l_{1}}}\left(n, \overline{\lambda^{1}}\right) \otimes L_{\widehat{\mathfrak{s l}_{l}}}\left(n, \overline{\lambda^{2}}\right) \otimes L_{\widehat{h_{\ell}}}\left(n, \lambda^{3}\right) \otimes \widetilde{M}\left(\lambda^{1}, \lambda^{2}, \lambda^{3}\right) \\
= & \bigoplus_{\lambda^{1}, \lambda^{2}, \lambda^{3}}\left[\bigoplus_{\gamma^{1} \in K_{1}^{\circ} / K_{1}} V_{K_{1}+\overline{\lambda^{1}}+\gamma^{1}} \otimes U^{\left(n, l_{1}\right)}\left(\overline{\lambda^{1}}, \gamma^{1}\right)\right] \otimes \\
& {\left[\bigoplus_{\gamma^{2} \in K_{2}^{\circ} / K_{2}} V_{K_{2}+\overline{\lambda^{2}}+\gamma^{2}} \otimes U^{\left(n, l_{2}\right)}\left(\overline{\lambda^{2}}, \gamma^{2}\right)\right] \otimes L_{\widehat{\mathfrak{h}}_{\underline{\ell}}}\left(n, \lambda^{3}\right) \otimes \widetilde{M}\left(\lambda^{1}, \lambda^{2}, \lambda^{3}\right), }
\end{aligned}
$$

where $L_{\widehat{\mathfrak{s l}}_{l_{i}}}\left(n, \overline{\lambda^{i}}\right)$ are irreducible $L_{\widehat{\mathfrak{s l}}_{l_{i}}}(n, 0)$-modules for $i=1,2$ and $L_{\widehat{\mathfrak{h}}_{\underline{\ell}}}\left(n, \lambda^{3}\right)$ is an irreducible $L_{\widehat{\mathfrak{h}}_{\underline{\ell}}}(n, 0)$-module with lowest weight $\lambda^{3} \in \mathbb{Q}_{+} \cdot M\left(\lambda^{1}, \lambda^{2}, \lambda^{3}\right)$ are modules of $C_{L_{\widehat{s} l_{l}}(n, 0)}\left(L_{\widehat{\mathfrak{l}_{\underline{e}}}}(n, 0)\right)$ and $\widetilde{M}(0,0,0)=C_{L_{\widehat{\mathfrak{s l} l}}(n, 0)}\left(L_{\widehat{\underline{\underline{\ell}}}}(n, 0)\right)=K\left(\mathfrak{s} l_{l}, \underline{\mathfrak{l}_{\underline{\ell}}}, n\right)$. So we have

$$
K\left(\mathfrak{s} \mathfrak{l}_{l}, n\right)=\bigoplus_{\lambda^{1}, \lambda^{2}} U^{\left(n, l_{1}\right)}\left(\overline{\lambda^{1}},-\overline{\lambda^{1}}\right) \otimes U^{\left(n, l_{2}\right)}\left(\overline{\lambda^{2}},-\overline{\lambda^{2}}\right) \otimes \widetilde{M}\left(\lambda^{1}, \lambda^{2}, 0\right) .
$$


By Theorem 3.10, we have

$$
K\left(\mathfrak{s l} \mathfrak{l}_{l}, n\right) \cong C_{L_{\mathfrak{s l n}_{n}}(1,0) \otimes l}\left(L_{\widehat{\mathfrak{s l}_{n}}}(l, 0)\right) .
$$

Let $\tau$ be the isomorphism from $C_{L_{\widehat{\mathfrak{s}_{n}}}(1,0) \otimes l}\left(L_{\widehat{\mathfrak{s l}_{n}}}(l, 0)\right)$ to $K\left(\mathfrak{s l}_{l}, n\right)$. Then

$$
\begin{aligned}
& \tau\left(\bigoplus_{\lambda \in P_{+}^{n, l_{1}}} \bigoplus_{\mu \in P_{+}^{n, l_{2}}} M^{\left(l_{1}, n\right)}(\bar{\lambda}) \otimes M^{\left(l_{2}, n\right)}(\bar{\mu}) \otimes M(\lambda, \mu, 0)\right) \\
& \quad=\bigoplus_{\lambda^{1}, \lambda^{2}} U^{\left(n, l_{1}\right)}\left(\overline{\lambda^{1}},-\overline{\lambda^{1}}\right) \otimes U^{\left(n, l_{2}\right)}\left(\overline{\lambda^{2}},-\overline{\lambda^{2}}\right) \otimes \widetilde{M}\left(\lambda^{1}, \lambda^{2}, 0\right)
\end{aligned}
$$

and

$$
\begin{aligned}
\tau\left(M^{\left(l_{1}, n\right)}(0)\right) & =U^{\left(n, l_{1}\right)}(0,0)=K\left(\mathfrak{s l}_{l_{1}}, n\right), \\
\tau\left(M^{\left(l_{2}, n\right)}(0)\right) & =U^{\left(n, l_{2}\right)}(0,0)=K\left(\mathfrak{s l}_{l_{2}}, n\right) .
\end{aligned}
$$

Considering $\bigoplus_{\lambda \in P_{+}^{n, l_{1}}} \bigoplus_{\mu \in P_{+}^{n, l_{2}}} M^{\left(l_{1}, n\right)}(\bar{\lambda}) \otimes M^{\left(l_{2}, n\right)}(\bar{\mu}) \otimes M(\lambda, \mu, 0)$ and $\bigoplus_{\lambda^{1}, \lambda^{2}} U^{\left(n, l_{1}\right)}\left(\overline{\lambda^{1}},-\overline{\lambda^{1}}\right) \otimes$ $U^{\left(n, l_{2}\right)}\left(\overline{\lambda^{2}},-\overline{\lambda^{2}}\right) \otimes \widetilde{M}\left(\lambda^{1}, \lambda^{2}, 0\right)$ as $K\left(\mathfrak{s l}_{l_{1}}, n\right) \otimes K\left(\mathfrak{s l}_{l_{2}}, n\right)$-modules and using (2) of Lemma 3.6 , we have

$$
\tau(M(0,0,0))=\widetilde{M}(0,0,0)
$$

That is,

$$
C_{L_{\widehat{\mathfrak{s l n}}}\left(l_{1}, 0\right) \otimes L_{\widehat{\mathfrak{s l n}}}\left(l_{2}, 0\right)}\left(L_{\widehat{\mathfrak{s l}} \widehat{l}_{n}}(l, 0)\right) \cong C_{L_{\widehat{\mathfrak{s l}} l}(n, 0)}\left(L_{\widehat{\mathfrak{s l}_{l_{1}}}}(n, 0) \otimes L_{\widehat{\mathfrak{s l} l_{2}}}(n, 0) \otimes L_{\widehat{\mathfrak{h}_{\ell}}}(n, 0)\right) .
$$

Corollary 4.3. For any $\underline{\ell}$ and $n, C_{L_{\mathfrak{s |}|\underline{\underline{\ell}}|}(n, 0)}\left(K\left(\mathfrak{s l}_{|\underline{\mid}|}, \underline{l}_{\underline{\ell}}, n\right)\right)$ is isomorphic to the tensor product of the vertex operator algebras corresponding the semi-simple Lie algebras and a lattice vertex operator algebra, and in particular is a rational vertex operator algebra.

Proof. One notes that $C_{L_{\mathfrak{s} \widehat{\mid} \mid \underline{l}}(n, 0)}\left(K\left(\mathfrak{s l}_{|\underline{\ell}|}, \mathfrak{l}_{\underline{\ell}}, n\right)\right) \supseteq L_{\widehat{\mathfrak{l}_{\underline{\ell}}}}(n, 0)$. Then by using (4.1) and (4.2) by setting $\left(\lambda^{1}, \lambda^{2}, \lambda^{3}\right)=(0,0,0)$, a similar argument to the proof of (3.4) and (3.5), with $n$ and $l$ switched, will prove the corollary. The vertex operator algebra corresponding to the semi-simple Lie algebra is $\left(\bigotimes_{k=1, l_{k} \geq 2}^{m} L_{\widehat{\mathfrak{s l}_{k}}}(n, 0)\right)$ which is rational. The lattice vertex operator algebra corresponds to the Heisenberg vertex operator algebra $L_{\widehat{h}_{\underline{\ell}}}(n, 0)$.

\section{Duality Pairs And Reciprocity}

5.1. Let $(V, Y, \mathbf{1}, \omega)$ be a vertex operator algebra. A semi-conformal vertex subalgebra of $V$ is a vertex subalgebra $U$ together with a conformal vector $\omega^{\prime} \in U$ such that $\left(U,\left.Y\right|_{U}, \mathbf{1}, \omega^{\prime}\right)$ is a vertex operator algebra and $\left.L(n)\right|_{U}=\left.L^{\prime}(n)\right|_{U}$ for all $n \geq-1$, where $L^{\prime}(n) \in \operatorname{End}(V)$ is defined by $Y\left(\omega^{\prime}, z\right)=\sum L^{\prime}(n) z^{-n-2}$. Note that if $\left.L(-2)\right|_{U}=\left.L^{\prime}(-2)\right|_{U}$, then $\omega=\omega^{\prime}$. In this case, $V$ is called a conformal extension of $U$ and $U$ is called a conformal vertex operator subalgebra. One can check the proof of [LL, Theorem 3.11.12] that if $U$ is a semi-conformal subalgebra of $V$, then $C_{V}(U)$ is also a semi-conformal vertex subalgebra of $V$ with conformal vector $\omega-\omega^{\prime}$. A semi-conformal subalgebra $\left(U, \omega^{\prime}\right)$ of $V$ is called closed if $C_{V}\left(C_{V}(U)\right)=U$. We will denote $\bar{U}=C_{V}\left(C_{V}(U)\right)$ if $V$ is understood 
from the context. If $U$ is closed in $V$, then $U=\operatorname{ker}\left(L(-1)-L^{\prime}(-1): V \rightarrow V\right)$ is uniquely determined by $\omega^{\prime}$. In fact the converse also holds.

Lemma 5.1. If $\left(U, \omega^{\prime}\right)$ and $\left(U, \omega^{\prime \prime}\right)$ are two semi-conformal subalgebras of $V$, then $\omega^{\prime}=\omega^{\prime \prime}$.

Proof. By definition we have $\left.L^{\prime}(n)\right|_{U}=\left.L(n)\right|_{U}=\left.L^{\prime \prime}(n)\right|_{U}$ for all $n \geq-1$. In particular, $\left(U, \omega^{\prime}\right)$ is a semi-conformal subalgebra of $\left(U, \omega^{\prime \prime}\right)$ and the vice verse. By [LL, Corollary 3.11.11], $C_{\left(U, \omega^{\prime}\right)}\left(U, \omega^{\prime \prime}\right)=\mathbb{C} 1$. By [LL, Theorem 3.11.12], $\omega^{\prime}-\omega^{\prime \prime} \in C_{\left(U, \omega^{\prime}\right)}\left(U, \omega^{\prime \prime}\right)$. So $\omega^{\prime}=\omega^{\prime \prime}$.

5.2. Let $U$ be a vertex operator algebra and $M$ a $V$-module. We denote by $\operatorname{Irr}_{M}(U)$ the set of isomorphism classes of irreducible $U$-modules $N$ such that $\operatorname{Hom}_{U}(N, M) \neq 0$. If $M$ is a semi-simple $U$-module, then we have

$$
M \cong \bigoplus_{N \in \operatorname{Irr}_{M}(U)} N \otimes \operatorname{Hom}_{U}(N, M)
$$

as $U$-modules. If $U$ is a semi-conformal subalgebra of a vertex operator algebra $V$ and $M$ is a $V$-module, then $\operatorname{Hom}_{U}(N, M)$ is a $C_{V}(U)$-module and is denoted as $\rho_{M}(N)$ if $U$ and $V$ are understood from the context. Let $V$ be a simple vertex operator algebra, a pair of semi-conformal simple vertex operator subalgebras $\left(U^{1}, U^{2}\right)$ of $V$ is called a duality pair in $V$ with respect to a $V$-module $M$ if (a) $U^{i}=C_{V}\left(U^{j}\right)$ with $i \neq j$ and (b)

$$
M=\bigoplus N^{1} \otimes \rho_{M}\left(N^{1}\right)
$$

as a $U^{1} \otimes U^{2}$-module. Here the summation is taken over all $N^{1} \in \operatorname{Irr}_{M}\left(U^{1}\right)$ of irreducible $U_{1}$-submodules such that $\operatorname{Hom}_{U^{1}}\left(N^{1}, M\right) \neq 0$ and $\rho_{M}: \operatorname{Irr}_{M}\left(U^{1}\right) \rightarrow \operatorname{Irr}_{M}\left(U^{2}\right)$ is a bijection.

In case $M=V$, we simply say that $\left(U^{1}, U^{2}\right)$ is a duality pair in $V$. This concept is motivated by the duality pairs of reductive groups defined by Howe in [Ho2]

Note that since both $U^{1}$ and $U^{2}$ are simple, $U^{1} \otimes U^{2}$ is a (conformal) vertex operator subalgebra of $V$. Also for any simple vertex operator algebra $V$, if one of $U^{i}$ is a lattice vertex operator algebra, then the rationality and the fusion rules of lattice vertex operators state in Theorem 2.8 imply that (b) is a consequence of (a) for all irreducible $V$-modules $M$. We now summarize the results from Section 3 and Section 4:

Lemma 3.2 and formula (3.5) implies that $C_{V}\left(C_{V}\left(V_{\mathfrak{h}_{n}}(l, 0)\right)\right)=V_{K}$. Hence $\left(V_{K}, V_{N_{n}^{l}}\right)$ is a duality pair in $V=L_{\widehat{\mathfrak{g}}}(1,0)^{\otimes l}=V_{A_{n-1}^{\times l}}$ with respective the irreducible module $V_{A_{n-1}^{\times l}}$. This is the case that when an even lattice $L=L^{\prime} \perp L^{\prime \prime}$ then $\left(V_{L^{\prime}}, V_{L^{\prime \prime}}\right)$ is a duality pair in $V_{L}$ with respect to $V_{L}$.

Theorem 3.10 implies that $\left(K\left(\mathfrak{s l}_{n}, l\right), K\left(\mathfrak{s l}_{l}, n\right)\right)$ is a duality pair in $V_{N_{n}^{l}}$ with respect to $V_{N_{n}^{l}}$.

Theorem 5.2. $\left(K\left(\mathfrak{s l}_{l}, n\right), V_{\sqrt{n} A_{l-1}}\right)$ is a duality pair in $L_{\widehat{\mathfrak{s l}_{l}}}(n, 0)$. 
By Theorem 3.10 and Theorem 5.2 we have

Corollary 5.3. $\left(L_{\widehat{\mathfrak{s l}}_{n}}(l, 0), K\left(\mathfrak{s l}_{l}, n\right)\right)$ is a duality pair in $L_{\widehat{\mathfrak{s l}}_{n}}(1,0)^{\otimes l}=V_{A_{n-1}^{\times l}}$.

We have the following chain of semi-conformal vertex subalgebras:

$$
V_{\sqrt{l} A_{n-1}} \subseteq L_{\mathfrak{s l n}_{n}}(l, 0) \subseteq L_{\mathfrak{s l}_{n}}\left(l_{1}, 0\right) \otimes \cdots \otimes L_{\mathfrak{s l}_{n}}\left(l_{s}, 0\right) \subseteq L_{\widehat{\mathfrak{s l}}_{n}}(1,0)^{\otimes l}=V_{A_{n-1}^{\times l}},
$$

where $l=l_{1}+\cdots+l_{s}$. Note that each of the vertex operator algebras is rational. Although the following two vertex operator algebras

$$
V_{\widehat{\mathfrak{h}}}(l, 0) \subseteq L_{\widehat{\widehat{\underline{\ell}}}_{\underline{\underline{e}}}}(l, 0)
$$

in $L_{\widehat{\mathfrak{s} \widehat{I}_{n}}}(l, 0)$ are not rational, the maximal conformal extensions $\bar{V}_{\widehat{\mathfrak{h}}}(l, 0) \subseteq \bar{L}_{\widehat{\mathfrak{l}}_{\underline{\ell}}}(l, 0)$ in $L_{\widehat{\mathfrak{s}}_{n}}(l, 0)$ are rational. The following discussions of duality pairs will use this fact.

Let $W=L_{\widehat{\mathfrak{s l n}}}(1,0)^{\otimes l}$ and $V=L_{\widehat{\mathfrak{s l n}_{n}}}\left(l_{1}, 0\right) \otimes \cdots \otimes L_{\widehat{\mathfrak{s l n}_{n}}}\left(l_{s}, 0\right)$ and $U=L_{\widehat{\mathfrak{s l n}_{n}}}(l, 0)$. We will study the following pairs in a sequel paper:

$$
\left(V, C_{W}(V)\right) \text { in } W, \quad\left(U, C_{V}(U)\right) \text { in } V, \quad\left(U, C_{W}(U)\right) \text { in } W .
$$

5.3. Let $\left(U^{1}, U^{2}\right)$ and $\left(U^{\prime 1}, U^{\prime 2}\right)$ be two duality pairs in $V$ with respect to an irreducible $V$-module $M$. We use $\rho_{M}: \operatorname{Irr}_{M}\left(U^{1}\right) \rightarrow \operatorname{Irr}_{M}\left(U^{2}\right)$ and $\rho_{M}^{\prime}: \operatorname{Irr}_{M}\left(U^{\prime 1}\right) \rightarrow \operatorname{Irr}_{M}\left(U^{\prime 2}\right)$ respectively to denote the correspondence. In case $U^{1} \subseteq U^{\prime 1}$, then these two duality pairs will have certain see and saw duality property. For reductive groups, various constructions of pairs of duality pairs are discussed in $[\mathrm{Ku}]$ and vertex operator algebra versions of these constructions are expected to carry over as well.

Theorem 5.4. If $U^{1} \subseteq U^{\prime 1}$, then $\left(U^{1}, U^{2} \cap U^{\prime 1}\right)$ is a duality pair in $U^{\prime 1}$ with respect to any $M^{\prime 1} \in \operatorname{Irr}_{M}\left(U^{\prime 1}\right)$ and $\left(U^{2} \cap U^{\prime 1}, U^{\prime 2}\right)$ is a duality pair in $U^{2}$ with respect to any $M^{2} \in \operatorname{Irr}_{M}\left(U^{2}\right)$.

Proof. By (a) of the duality pair condition, $U^{1} \subseteq U^{\prime 1}$ implies that $U^{2} \supseteq U^{\prime 2}$. Thus $U^{\prime 1} \cap U^{2}=U^{\prime 1} \cap C_{V}\left(U^{1}\right)=C_{U^{\prime 1}}\left(U^{1}\right)$. Similarly, $U^{1}=C_{V}\left(U^{2}\right)=U^{\prime 1} \cap C_{V}\left(U^{2}\right)=$ $C_{U^{\prime 1}}\left(U^{\prime 1} \cap U^{2}\right)$. Thus the condition (a) holds for the pair $\left(U^{1}, U^{\prime 1} \cap U^{2}\right)$. To check the condition (b), we first have, for each $M^{1} \in \operatorname{Irr}_{M}\left(U^{1}\right)$,

$$
\operatorname{Hom}_{U^{1}}\left(M^{1}, M\right)=\oplus_{M^{\prime 1} \in \operatorname{Irr}_{M}\left(U^{\prime 1}\right)} \operatorname{Hom}_{U^{1}}\left(M^{1}, M^{\prime 1}\right) \otimes \rho_{M}^{\prime}\left(M^{\prime 1}\right)
$$

as $U^{2}$-modules. Thus we get the following decomposition of $M$ as $U^{1}$-modules,

$$
M=\bigoplus_{M^{1} \in \operatorname{Irr}_{M}\left(U^{1}\right)} \bigoplus_{M^{\prime 1} \in \operatorname{Irr}_{M}\left(U^{\prime 1}\right)} M^{1} \otimes \operatorname{Hom}_{U^{1}}\left(M^{1}, M^{\prime 1}\right) \otimes \rho_{M}^{\prime}\left(M^{\prime 1}\right) .
$$

Now comparing with the decomposition of $M$ as $U^{\prime 1}$-modules

$$
M=\bigoplus_{M^{\prime 1} \in \operatorname{Irr}_{M}\left(U^{\prime 1}\right)} M^{\prime 1} \otimes \rho_{M}^{\prime}\left(M^{\prime 1}\right)
$$


and by applying the functor $M^{\prime 1}=\operatorname{Hom}_{U^{\prime 2}}\left(\rho_{M}^{\prime}\left(M^{\prime 1}\right), M\right)$ to get

$$
M^{\prime 1}=\bigoplus_{M^{1} \in \operatorname{Irr}_{M}\left(U^{1}\right)} M^{1} \otimes \operatorname{Hom}_{U^{1}}\left(M^{1}, M^{\prime 1}\right)
$$

which is an isomorphism of $U^{\prime 1}$-modules. Note that $M^{1} \in \operatorname{Irr}_{M^{\prime 1}}\left(U^{1}\right)$ if and only if $\operatorname{Hom}_{U^{1}}\left(M^{1}, M^{\prime 1}\right) \neq 0$. Thus we have the bijection $\operatorname{Irr}_{M^{\prime 1}}\left(U^{1}\right) \rightarrow \operatorname{Irr}_{M^{\prime 1}}\left(U^{2} \cap U^{\prime 1}\right)$. The other duality pair follows from a similar argument by replacing the pairs $U^{1} \subseteq U^{\prime 1}$ with $U^{\prime 2} \subseteq U^{2}$.

In the above proof, we notice that $U^{\prime 2} \subseteq U^{2}$. Hence any $U^{2}$-module $\rho_{M}\left(M^{1}\right)$ is a $U^{\prime 2}$-module. Thus we have

Corollary 5.5. With the assumption of the theorem, for any $M^{1} \in \operatorname{Irr}_{M}\left(U^{1}\right)$ and $M^{1} \in$ $\operatorname{Irr}_{M}\left(U^{\prime 1}\right)$, we have an isomorphism of $U^{2} \cap U^{\prime 1}$-modules

$$
\operatorname{Hom}_{U^{1}}\left(N, N^{\prime}\right) \cong \operatorname{Hom}_{U^{\prime 2}}\left(\rho_{M}^{\prime}\left(N^{\prime}\right), \rho_{M}(N)\right)
$$

This isomorphism provides a reciprocity law for vertex operator algebras in the sense of [Ho2] which was for reductive groups.

5.4. We now apply reciprocity law in the above corollary to various cases we discussed in this paper.

(1) In $L_{\widehat{\mathfrak{s l}} \underline{l}}(n, 0)$, we consider the two duality pairs $\left(U^{\prime 1}, U^{\prime 2}\right)=\left(\bar{L}_{\widehat{\mathfrak{l}}_{\underline{l}}}(n, 0), K\left(\mathfrak{s l}_{l}, \mathfrak{l}_{\underline{\ell}}, n\right)\right)$ and $\left(U^{1}, U^{2}\right)=\left(V_{\sqrt{n} A_{l-1}}, K\left(\mathfrak{s l}_{l}, n\right)\right)$. Then we have isomorphisms

$$
C_{\bar{L}_{\widehat{\underline{\underline{e}}}_{\underline{l}}}(n, 0)}\left(V_{\sqrt{n} A_{l-1}}\right) \cong C_{K\left(\mathfrak{s}_{l}, n\right)}\left(K\left(\mathfrak{s \mathfrak { l } _ { l }}, \mathfrak{l}_{\underline{\ell}}, n\right)\right)
$$

which is isomorphic further to $K\left(\mathfrak{s l}_{l}, n\right) \cap \bar{L}_{\widehat{\mathfrak{l}_{\underline{\ell}}}}(n, 0)$ for any $\underline{\ell}=\left(l_{1}, \cdots, l_{s}\right)$.

(2) Similarly if we apply the corollary to the two duality pairs $\left(U^{1}, U^{2}\right)=\left(V_{\sqrt{l} A_{n-1}}, V_{N_{n}^{l}}\right)$ and $\left(U^{\prime 1}, U^{\prime 2}\right)=\left(L_{\widehat{\mathfrak{s l}_{n}}}(l, 0), K\left(\mathfrak{s l}_{l}, n\right)\right)$ in $V_{A_{n-1}^{\times l}}$ with respective to $M=V_{A_{n-1}^{\times l}}$. We get

$$
K\left(\mathfrak{s l}_{n}, l\right)=C_{L_{\widehat{s} l_{n}}(l, 0)}\left(V_{\sqrt{l} A_{n-1}}\right) \cong C_{V_{N_{n}^{l}}}\left(K\left(\mathfrak{s l}_{l}, n\right)\right)
$$

which follows from the proof of Theorem 3.10.

(3) Now let us set $\left(U^{1}, U^{2}\right)=\left(L_{\widehat{\mathfrak{s l}}_{n}}(l, 0), K\left(\mathfrak{s l}_{l}, n\right)\right)$ and $\left(U^{\prime 1}, U^{\prime 2}\right)=\left(L_{\widehat{\mathfrak{s l}}_{n}}\left(l_{1}, 0\right) \otimes \cdots \otimes\right.$ $\left.L_{\widehat{s i}_{n}}\left(l_{s}, 0\right), K\left(\mathfrak{s l}_{l_{1}}, n\right) \otimes \cdots \otimes K\left(\mathfrak{s l}_{l_{s}}, n\right)\right)$ in $V_{A_{n-1}^{\times l}}$ with respective to $M=V_{A_{n-1}^{\times l}}$. Here we use the convention that $K\left(\mathfrak{s l}_{1}, n\right)=\mathbb{C} 1$. Then by Theorem 4.2 we get

$$
K\left(\mathfrak{s l}_{l}, \mathfrak{l}_{\underline{\ell}}, n\right) \cong C_{L_{\widehat{\mathfrak{s l n}}}\left(l_{1}, 0\right) \otimes \cdots \otimes L_{\widehat{\mathfrak{s l n}}}\left(l_{s}, 0\right)}\left(L_{\widehat{\mathfrak{s l}}_{n}}(l, 0)\right) \cong C_{K(\mathfrak{s l} l, n)}\left(K\left(\mathfrak{s l}_{l_{1}}, n\right) \otimes \cdots \otimes K\left(\mathfrak{s l} l_{l}, n\right)\right)
$$




\section{Applichtions and Examples}

In this section we summarize known results on the question of rationality of the coset construction and parafermions using Theorem 1.1.

The structure of parafermion vertex operator algebras has been studied extensively recently ( see [ALY, DLWY, DLY2, DW1, DW2] etc.). Most of the results concentrated on $K\left(\mathfrak{s l}_{2}, l\right)$. The next result gives rationality of parafermion vertex operator algebras for all $\mathfrak{s l}_{n}$ in the level 2 case.

Theorem 6.1. For any $n \geq 2$, the parafermion $K\left(\mathfrak{s l}_{n}, 2\right)$ is always rational and it has $2^{n-2}(n+1)$ irreducible representations.

Proof. By Theorem 1.1, $K\left(\mathfrak{s l}_{n}, 2\right) \cong C_{L_{\mathfrak{s l}_{2}}(1,0)^{\otimes n}}\left(L_{\widehat{\mathfrak{s l}_{2}}}(n, 0)\right)$. Now the theorem follows from [JL, Thm. 4.16, Thm. 5.5].

Conversely the known results of parafermions can be transferred to coset constructions.

Theorem 6.2. (1) $C_{L_{\widehat{\mathfrak{s}_{n}}}(1,0) \otimes l}\left(L_{\widehat{\mathfrak{s}_{n}}}(l, 0)\right)$ is always $C_{2}$-cofinite.

(2) The Zhu algebra $A\left(C_{L_{\widehat{s} \Gamma_{n}}(1,0) \otimes 2}\left(L_{\widehat{\mathfrak{s l n}_{n}}}(2,0)\right)\right)$ is semisimple, commutative, of dimension $n(n+1) / 2$. In particular, $C_{L_{\widehat{s} I_{n}}(1,0)^{\otimes 2}}\left(L_{\widehat{\mathfrak{s l n}_{n}}}(2,0)\right)$ has exactly $n(n+1) / 2$ non-isomorphic irreducible representations.

(3) $C_{L_{\widehat{s ! n}}(1,0)^{\otimes 2}}\left(L_{\widehat{\mathfrak{s l n}_{n}}}(2,0)\right)$ is rational for $n \leq 6$.

Proof. (1) follows from [ALY, Thm. 10.5]. (2) follows from [ALY, Thm. 8.2] and [DLY2] and (3) follows from [DLY2].

\section{REFERENCES}

[A] T. Abe, Fusion rules for the charge conjugation orbifold, J. Alg. 242 (2001), 624-655.

[ADL] T. Abe, C. Dong and H. Li, Fusion rules for the vertex operator $M(1)^{+}$and $V_{L}^{+}$, Comm. Math. Phys. 253 (2005), 171-219.

[ABI] D. Altschüler, M. Bauer, and C. Itzykson, The Branching Rules of Conformal Embeddings, Commun. Math. Phys. 132 (1990), 349-364.

[ABS] D. Altschüler, M. Bauer, and H. Saleur, Level-rank duality in non-unitary coset theories, J. Phys. A: Math. Gen.23 (1990), L789-L793.

[AS] D. Altschüler and H. Saleur, Level-rank duality in quantum groups, Nuclear Physics B $\mathbf{3 5 4}$ (1991), 579-613.

[ALY] T. Arakawa, C. Lam and H. Yamada, Zhu's algebra, $C_{2}$-algebras and $C_{2}$-cofiniteness of parafermion vertex operator algebras, arXiv: 1207.3909vl.

[BEHHH] R. Blumenhagen, W. Eholzer, A. Honecker, K. Hornfeck and R. Hübel, Coset realization of unifying $W$-algebras, Internat. J. Modern Phys. A10 (1995), 2367-2430.

[B] R. E. Borcherds, Vertex algebras, Kac-Moody algebras, and the Monster, Proc. Natl. Acad. Sci. USA 83 (1986), 3068-3071. 
[CGT] A. Cappelli, L. S. Georgiev and I. T. Todorov, Parafermion Hall states from coset projections of abelian conformal theories, Nucl. Phys. B599 (2001), 499C530.

[CL] T. Chen and C. Lam, Extension of the tensor product of unitary Virasoro vertex operator algebra, Commun. Alg. 35 (2007), 2487-2505.

[CLW] J. Chen, N. Lam, and W. Wang, Super duality and irreducible characters of ortho-symplectic Lie superalgebras, Invent. Math. 183 (2011) 189-224.

[Dix] J. Dixmier, Enveloping algebras Graduate Studies in Mathematics, 11. American Mathematical Society, Providence, RI, 1996

[D] C. Dong, Representations of the moonshine modulevertex operator algebra, Contemp. Math. 175 (1994), 27-36.

[DGH] C. Dong, R. Griess Jr. and G. Hoehn, Framed vertex operator algebras, codes and the moonshine module, Commun. Math. Phys. 193 (1998), 407-448.

[DJL] C. Dong, C. Jiang and X. Lin, Rationality of vertex operator algebra $V_{L}^{+}$: higher rank, Proc. Lond. Math. Soc. 104 (2012), 799-826.

[DLWY] C. Dong, C. Lam, Q. Wang and H. Yamada, The structure of parafermion vertex operator algebras, J. Alg. 323 (2010), 371-381.

[DLY1] C. Dong, C. Lam and H. Yamada, Decomposition of the vertex operator algebra $V_{\sqrt{2} A_{3}}, J$. Alg. 222 (1999), 500-510.

[DLY2] C. Dong, C. Lam and H. Yamada, $W$-algebras related to parafermion vertex operator algebras, J. Alg. 322 (2009), 2366-2403.

[DL] C. Dong and J. Lepowsky, Generalized vertex algebras and relative vertex operators, Progress in Math., Vol. 112, Birkhäuser, Boston, 1993.

[DLM1] C. Dong, H. Li and G. Mason, Regularity of rational vertex operator algebras, Adv. Math.132 (1997), 148-166.

[DLM2] C. Dong, H. Li and G. Manson, Simple currents and extensions of vertex operator algebras, Commun. Math. Phys. 180 (1996), 671-707.

[DLM3] C. Dong, H. Li and G. Mason, Twisted representations of vertex operator algebras, Math. Ann. 310 (1998), 571-600.

[DLM4] C. Dong, H. Li and G. Mason, Modular invariance of trace functions in orbifold theory and generalized moonshine. Comm. Math. Phys. 214 (2000), 1-56.

[DLMN] C. Dong, H. Li, G. Mason and S. P. Notton, Associative subalgebras of the Griess algebra and related topics, in "The Monster and Lie algebras" (J. Ferrar and K. Harada, Eds.) de Gruyter, Berlin, 1998, pp. 27-42.

[DMZ] C. Dong, G. Mason and Y. Zhu, Discrete series of the Virasoro algebra and the moonshine module, Proc. Symp. Pure. Math. American Math. Soc. 56 II (1994), 295-316.

[DW1] C. Dong and Q. Wang, The structure of parafermion vertex operator algebras: general case, Commun. Math. Phys. 299 (2010), 783-792.

[DW2] C. Dong and Q. Wang, On $C_{2}$-cofiniteness of parafermion vertex operator algebras, J. Alg. 328 (2011), 420-431.

[DW3] C. Dong and Q. Wang, Parafermion vertex operator algebras, Front. Math. China , 6 (2011), 567-579.

[FF] B. Feigin and D. Fuchs, Verma modules over the Virasoro algebra, Topology (Leningrad, 1982), Lecture Notes in Math. 1060. Berlin-New York, Springer: 230-245.

[Fr] I. B. Frenkel, Representations of affine Lie algebras, Hecke modular forms and Korteweg-de Vries type equations. Lie algebras and related topics (New Brunswick, N.J., 1981) pp. 71-110, Lecture Notes in Math., 933, Springer, Berlin-New York, 1982. 
[FHL] I. B. Frenkel, Y. Huang and J. Lepowsky, On axiomatic approaches to vertex operator algebras and modules, Memoirs American Math. Soc. 104, 1993.

[FLM] I. B. Frenkel, J. Lepowsky and A. Meurman,Vertex Operator Algebras and the Monster, Pure and Applied Math. Vol. 134, Academic Press, 1988.

[FZ] I. B. Frenkel and Y. Zhu, Vertex operator algebras associated to representations of affine and Virasoro algebras, Duke Math. J. 66 (1992), 123-168.

[GQ] D. Gepner and Z. Qiu, Modular invariant partition functions for parafermionic field theories, Nucl. Phys. B285 (1987), 423-453.

[GKO1] P. Goddard, A. Kent and D. Olive, Virasoro algebras and coset space models, Phys. Lett. B152 (1985), 88-92.

[GKO2] P. Goddard, A. Kent and D. Olive, Unitary representations of the Virasoro and super-Virasoro algebra, Commun. Math. Phys. 103 (1986), 105-119.

[Ho1] R. Howe, Perspectives on invariant theory: Schur duality, multiplicity-free actions, and beyond, The Schur Lectures (1992), Israel Math. Conf. Proceedings, 8 (1995), 1-182.

[Ho2] R. Howe Reciprocity law in the theory of duality pairs, Representation theory of reductive groups (Park City, Utah, 1982), pp. 159-175, Progr. Math., 40, Birkhäuser Boston, Boston, MA, 1983.

[HTW] R. Howe, E. Tan, and J. Willenbring, Reciprocity algebras and branching for classical symmetric pairs., pp 191-231 in Groups and analysis. The legacy of Hermann Weyl. Edited by Katrin Tent. London Mathematical Society Lecture Note Series, 354. Cambridge University Press, Cambridge, 2008.

[JL] C. Jiang and Z. Lin, The commutant of $L_{\widehat{\mathfrak{s l}}_{2}}(n, 0)$ in the vertex operator algebra $L_{\widehat{\mathfrak{s l}_{2}}}(1,0)^{\otimes n}$, arXiv: 1311.0608

[K] V. G. Kac, Infinite-dimensional Lie Algebras, 3rd ed., Cambridge Univ. Press, Cambridge, 1990.

[KR] V. G. Kac and A. Raina, Highest Weight Representations of Infinite Dimensional Lie Algebras, World Scientific, Adv. Ser. In Math. Phys., Singapore, 1987.

[KW] V. Kac and W. Wang, Vertex operator superalgebras and their representations, Contemp. Math. Amer. Math. Soc. 175 (1994), 161-191.

[KL] Y. Kawahigashi and R. Longo, Classification of local conformal nets. Case $c<1$, Ann. of Math. 160 (2004), 493-522.

[KLY] M. Kitazume, C. Lam and H. Yamada, Decomposition of the moonshine vertex operator algebra as Virasoro modules, J. Alg. 226 (2000), 893-919.

[KMY] M. Kitazume, M. Miyamoto and H. Yamada, Ternary codes and vertex operator algebras, J. Alg. 223 (2000), 379-395.

[Ku] S. Kudla, Seesaw dial reductive pairs, Automorphic forms of several variables (1984), 244-268.

[LL] J. Lepowsky and H. Li, Introduction to Vertex Operator Algebras and Their Representations, Progress in Mathematics, Vol. 227, Birkh user Boston, Inc., Boston, MA, 2004.

[L] H. Li, Some finiteness properties of regular vertex operator algebras, J. Alg. 212 (1999), 495-514.

[LS] C. Lam and S. Sakuma, On a class of vertex operator algebras having a faithful $S_{n+1}$-action, Taiwanese Journal of Mathematics, 12 (2008), 2465-2488.

[LY] C. Lam and H. Yamada, Decomposition of the lattice vertex operator algebra $V_{\sqrt{2} A_{l}}, J$. Alg. 272 (2004), 614-624.

[LZ] G. Lehrer, R. Zhang, The second fundamental theorem of invariant theory for the orthogonal group. Ann. Math. (2) 176 (2012), no. 3, 2031-2054.

[MO] A. Marian and D. Oprea, The level-rank duality for non-abelian theta functions. Invent. Math. 168 (2007), no. 2, 225-247.

$[\mathrm{MNT}]$ A. Matsuo, K. Nagatomo and A. Tsuchiya, Quasi-finite algebras graded by Hamiltonian and vertex operator algebras, math. QA/0505071. 
[M] M. Miyamoto, Representation theory of code vertex operator algebra, J. Algebra 201 (1998), $115-150$.

[MT] M. Miyamoto and K. Tanabe, Uniform product of $A_{g, n}(V)$ for an orbifold model $V$ and $G$-twisted Zhu algebra, J. Alg. 274 (2004), 80-96.

$[\mathrm{Mu}] \quad$ S. Mukhopadhyay, Rank-level duality and the conformal block divisors, arXiv:1308.0854v1

[NS] S. G. Naculich,and H. J. Schnitzer, Level-rank duality of the U(N) WZW model, Chern-Simons theory, and 2d qYM theory, J. High Energy Phys. 06023 (2007), 18 pp. (electronic).

[OS] V. Ostrik and M. Sun, Level-rank duality via tensor categories, arXiv:1208.5131v2, May 23, 2013.

[X1] F. Xu, Algebraic coset conformal field theories II, Publ. Res. Inst. Math. Sci. 35 (1999), 795-824.

[X2] F. Xu, Algebraic coset conformal field theories, Commnu. Math. Phys. 211 (2000), 1-43.

$[\mathrm{ZF}] \quad$ A. B. Zamolodchikov and V. A. Fateev, Nonlocal (parafermion) currents in two-dimensional conformal quantum field theory and self-dual critical points in ZN-symmetric statistical systems, Sov. Phys. JETP 62 (1985), 215-225.

[W] W. Wang, Rationality of Virasoro vertex operator algebras, Internat. Math. Res. Notices, 7 (1993), 197-211.

[Weyl] H. Weyl, The classical groups. Their invariants and representations. Fifteenth printing. Princeton Landmarks in Mathematics. Princeton University Press, Princeton, NJ, 1997.

[Z] Y. Zhu, Modular invariance of characters of vertex operator algebras, J. Amer, Math. Soc. 9 (1996), 237-302.

(Jiang) Department of Mathematics, Shanghai Jiaotong University, Shanghai, 200240, CHINA

E-mail address: cpjiang@sjtu.edu.cn

(Lin) Department of Mathematics, Kansas State University, Manhattan, KS 66506, USA

E-mail address: zlin@math.ksu.edu 